\title{
Pricing American Options Using a Nonparametric Entropy Approach
}

\author{
Xisheng Yu ${ }^{1}$ and Li Yang ${ }^{2}$ \\ ${ }^{1}$ School of Economic Mathematics, Southwestern University of Finance and Economics, Chengdu 611130, China \\ ${ }^{2}$ Australian School of Business, University of New South Wales, NSW 2052, Australia \\ Correspondence should be addressed to Xisheng Yu; yuxisheng@swufe.edu.cn
}

Received 4 February 2014; Accepted 7 March 2014; Published 8 May 2014

Academic Editor: Fenghua Wen

Copyright (c) $2014 \mathrm{X}$. Yu and L. Yang. This is an open access article distributed under the Creative Commons Attribution License, which permits unrestricted use, distribution, and reproduction in any medium, provided the original work is properly cited.

\begin{abstract}
This paper studies the pricing problem of American options using a nonparametric entropy approach. First, we derive a general expression for recovering the risk-neutral moments of underlying asset return and then incorporate them into the maximum entropy framework as constraints. Second, by solving this constrained entropy problem, we obtain a discrete riskneutral (martingale) distribution as the unique pricing measure. Third, the optimal exercise strategies are achieved via the leastsquares Monte Carlo algorithm and consequently the pricing algorithm of American options is obtained. Finally, we conduct the comparative analysis based on simulations and IBM option contracts. The results demonstrate that this nonparametric entropy approach yields reasonably accurate prices for American options and produces smaller pricing errors compared to other competing methods.
\end{abstract}

\section{Introduction}

According to modern asset pricing theory, the value of any asset can be calculated as the expectation under the riskneutral measure of discounted future cash flows. One of the challenging tasks in applying modern asset pricing theory to value options is to find an appropriate risk-neutral pricing measure. The maximum entropy principle is regarded as a reasonable criterion for determining an appropriate riskneutral pricing measure (see, e.g., Frittelli [1] and Stutzer [2]) because it maximizes the use of prior knowledge in obtaining the posterior distribution, while being maximally uninformative about missing or unknown information (Jaynes [3]).

Stutzer [2] is the first to propose a nonparametric entropy valuation method, named the canonical valuation method, for valuing European options. This method does not need to make any preassumption for the underlying asset and relies only upon historical underlying price data. In this nonparametric pricing method, the physical distribution is transformed to a posterior distribution under the principle of the maximum entropy framework by imposing the martingale constraint. Because of the martingale constraint, this posterior distribution can be considered as a riskneutral distribution (RND) of the underlying asset return and is then used to price the European options. The entropy valuation approach relies more on the information contained in market prices and less on normative assumptions. Due to this appealing feature, the entropy pricing method has been extended to price American options. Liu [4] proposed a socalled canonical least-squares Monte Carlo (CLM) method for pricing American options which uses Stutzer's framework to get the canonical distribution as pricing measure and determines the optimal exercise strategy via least-squares Monte Carlo algorithm (Longstaff and Schwartz [5]). Due to the sole martingale constraint used in CLM method, however, a problem arises if the martingale constraint does not sufficiently restrict the feasible set of measures to enable the entropy pricing measure to be close enough to the correct martingale measure.

Stutzer suggests that adding additional constraints forcing a subset of options to be priced correctly which would shrink the feasible set more tightly around the correct 
martingale measure. Following this, Alcock and Auerswald [6] incorporate the option price constraint, that is, choosing an option following certain criteria and forcing it to be priced correctly, into the entropy framework, in empirically pricing American options. This extended method utilizes the information individually and only the information contained in that specific option is extracted and incorporated into the entropy framework. Thus, it could only be accurate to price the option with the same characteristics such as the same strike price and the same time to expiration. Therefore, more option price constraints have to be put into the framework in order to incorporate more information for obtaining a better estimate of RND. But it may be computationally impossible due to the well-known problem that Jacobian matrix required in the estimation of the RND (see (12) and (13)) is singular or perhaps ill-conditioned (Agmon et al. [7] and Buchen and Kelly [8]), as the number of option price constraints increase.

It is well known that option prices contain information about market participants' perceptions of the distribution of the underlying asset ${ }^{1}$. Hence, information from the option market that characterizes the asset return distribution such as volatility, skewness, and kurtosis could also be considered as additional constraints and incorporated into the entropy framework. Indeed the moments can accurately characterize the shape of the underlying distribution, for instance, a normal distribution can be identified using the first- and second-order moments. The mentioned entropybased valuation approaches above ignore the significance of inferring the RND by using more useful information from option data and do not create any informative constraint about the moments of the underlying asset returns.

Due to those described above, this paper introduces an ideally extended entropy method, named the riskneutral moments-constrained entropic least-squares method (RMEL), by incorporating the risk-neutral moments (RNMs) as constraints for pricing American options. It is simple, general, and suitable for pricing most type of options such as path-dependent options. The key component that differentiates the RMEL method to the methods discussed above is the way that the information contained in option markets is exploited and utilized for recovering the risk-neutral pricing measure. As a result, our approach allows us to learn much more about the shape of RND. This proposed RMEL method does not need to impose preassumptions on either the market structure (e.g., completeness) or the process of underlying asset price, further, extracting moments are model-free and forward-looking. In addition, our approach can flexibly deal with the issues of dividends and time-varying interest rates.

Our valuation method proceeds in three stages. First, we estimate the noncentral RNMs of the underlying asset return from a set of American options based on the formulas we derive using a characteristic function. These RNMs not only guarantee the discounted price process of the underlying asset to be a martingale but also take the effects of the volatility smile, as well as the skewness and kurtosis of the asset returns, into account when generating a risk-neutral distribution. Second, we transform the physical distribution to the RND by maximizing the entropy value subject to RNM constraints estimated in the first stage. As there is no closed-form solution, we use the Nelder-Mead simplex numerical method to estimate the RND. We then generate the risk-neutral underlying price paths drawn directly from the estimated RND. Third, the value of American option is calculated by incorporating the least-squares Monte Carlo (LSM) algorithm to determine an optimal exercise strategy and then averaging the discounted expected payoffs along every path. As the generated paths are under the riskneutral measure, the discount rate is the same as the risk-free rate.

We evaluate the usefulness of our method and compare its performance in a number of ways with that of Liu [4] in the same simulation setting, and with that of Liu [4] and Alcock and Auerswald [6] as well as other benchmarks using IBM option contracts. The simulation results suggest that, with simulated returns from geometric Brownian motion (GBM), RMEL approach produces very similar prices for calls and puts as those of Black-Scholes and finite difference and outperforms the method of Liu [4] under the simulated environment. The empirical investigation also demonstrates that our valuation approach generates smaller pricing errors than those of Liu [4], Alcock and Auerswald [6], and other benchmark methods for pricing IBM options.

The remainder of this paper is organized as follows. Section 2 presents our entropy valuation framework with detailed procedures. Sections 3 and 4 compare our valuation technique with other approaches in a simulated environment and using IBM options, respectively. Section 5 presents our conclusions and remarks.

\section{RNMs-Constrained Entropy Valuation Method}

We present the risk-neutral moments-constrained entropic least-squares valuation approach by employing the RNMs constraints under the entropy pricing framework to price American options. First, the RNMs are estimated using a set of call options based on the formulas we derive via a characteristic function. We then replace the single martingale constraint in the entropic pricing framework with the estimated RNMs to generate a better estimate of the RND that takes into account not only the mean of the distribution but also its entire shape. Note that our first and second RNM constraints ensure the martingale property and consider the volatility smile effect. Given the RND, an independent random sample of future underlying returns is drawn to generate risk neutral price paths. Potential exercise points for American options are then determined along every sample path via the LSM algorithm proposed by Longstaff and Schwartz [5]. Finally, option prices are computed as the expectation of the discounted payoffs along the risk-neutral underlying paths. Further details of our approach are discussed in the following sections. 
2.1. Recovering RNMs from the Option Market. To estimate a RND of underlying asset returns more accurately, we incorporate RNMs as constraints into an entropic pricing framework. We recover RNMs from the option market, since it is well established in the literature that the option market contains useful information about the future return distributions of underlying assets ${ }^{2}$. In this way, volatility smile, which is commonly observed from the option market and skewness and excess kurtosis, can be effectively reflected in the estimation of the RND.

We first introduce some notations. Denote the price of the underlying asset at time $t$ by $S_{t}$. Let the $\tau$-period asset gross return at time $t$ be given by the price relative, $R_{t, \tau}=S_{t+\tau} / S_{t}$, and let the $\tau$-period $j$ th-order RNM at time $t, m_{t, \tau}(j)$, be defined as $m_{t, \tau}(j)=E_{\pi^{*}}\left(\left[\log \left(R_{t, \tau}\right)\right]^{j}\right)$, where the symbol $E_{\pi^{*}}$ represents the expectation operator under the risk-neutral probability measure $\pi^{*}$. Here, $\tau$ can be any appropriate time period such as a day or an hour. A special case is where $\tau$ is the period from time $t$ to the option expiration time $T$. In this case of $\tau=(T-t)$, the $j$ th-order RNM at time $t$ can be expressed as $m_{t, T-t}(j)=E_{\pi^{*}}\left(\left[\log \left(R_{t, T-t}\right)\right]^{j}\right)$. The relation between $m_{t, \tau}(j)$ and $m_{t, T-t}(j)$ is given in Theorem 3.

According to Bakshi et al. [9], $m_{t, T-t}(j)(j=1,2,3,4)$ can be recovered from a set of cross-sectional out-of-themoney (OTM) European options. The following lemma holds.

Lemma 1. Under the martingale pricing measures $\pi^{*}$, $m_{t, T-t}(j)$ can be recovered from the market prices of OTM European calls and put as follows.

The $(T-t)$-period first-order RNM $m_{t, T-t}(1)$ is given by

$$
\begin{aligned}
m_{t, T-t}(1)= & e^{(r-q)(T-t)} \\
& -e^{r(T-t)}\left[\int_{S_{t}}^{\infty} \frac{1}{K^{2}} C_{t}^{E}(T ; K) d K\right. \\
& \left.\quad+\int_{0}^{S_{t}} \frac{1}{K^{2}} P_{t}^{E}(T ; K) d K\right]-1 .
\end{aligned}
$$

The $(T-t)$-period $j$ th-order $\operatorname{RNM} m_{t, T-1}(j)(j \geq 2)$ is given by

$$
\begin{aligned}
& m_{t, T-t}(j)=j e^{r(T-t)}\left[\int_{S_{t}}^{\infty} \frac{(j-1)-\ln \left(K / S_{t}\right)}{K^{2}}\right.\left.\times\left[\ln \left(\frac{K}{S_{t}}\right)\right]^{(j-2)} C_{t}^{E}(T ; K) d K\right] \\
&+j e^{r(T-t)}\left[\int_{0}^{S_{t}} \frac{(j-1)-\ln \left(K / S_{t}\right)}{K^{2}}\right. \\
&\left.\quad \times\left[\ln \left(\frac{K}{S_{t}}\right)\right]^{(j-2)} P_{t}^{E}(T ; K) d K\right],
\end{aligned}
$$

where $C_{t}^{E}(T ; K)$ and $P_{t}^{E}(T ; K)$ are the prices of European call and put options at time $t$ with expiration time $T$ and strike price $K, r$ is the continuously compounded risk-free interest rate matching time to the option expiration, and $q$ is the continuously compounded dividend yield. Both $r$ and $q$ are annualized and assumed to be constant over time.

Proof. Provided in Appendix A ${ }^{3}$.

Lemma 1 provides formulas to extract the first four moments, which are related to the mean, volatility, skewness, and kurtosis of the risk-neutral return density from a set of OTM European calls and puts. We intend to recover the RNMs using American calls supposed not to be exercised prior to expiration ${ }^{4}$ and denote the price of American call option with expiration $T$ and strike price $K$ by $C_{t}^{A}(T ; K)$; then we have the following.

Corollary 2. When the call options are not being exercised prior to expiration, $m_{t, T-t}(j)$ can be recovered from American calls.

The $(T-t)$-period first-order RNM $m_{t, T-t}(1)$ is

$$
\begin{array}{r}
m_{t, T-t}(1)=e^{(r-q)(T-t)}-e^{r(T-t)}\left[\int_{S_{t}}^{\infty} \frac{1}{K^{2}} C_{t}^{A}(T ; K) d K\right] \\
-e^{r(T-t)}\left[\int _ { 0 } ^ { S _ { t } } \frac { 1 } { K ^ { 2 } } \left[C_{t}^{A}(T ; K)+K e^{-r(T-t)}\right.\right. \\
\left.\left.-S_{t} e^{-q(T-t)}\right] d K\right]-1 .
\end{array}
$$
by

The $(T-t)$-period jth-order $R N M m_{t, T-t}(j)(j \geq 2)$ is given

$$
\begin{gathered}
m_{t, T-t}(j) \\
=j e^{r(T-t)} \times\left[\int_{S_{t}}^{\infty} \frac{(j-1)-\ln \left(K / S_{t}\right)}{K^{2}}\right. \\
\left.\times\left[\ln \left(\frac{K}{S_{t}}\right)\right]^{(j-2)} C_{t}^{A}(T ; K) d K\right] \\
+j e^{r(T-t)} \times\left[\int_{0}^{S_{t}} \frac{(j-1)-\ln \left(K / S_{t}\right)}{K^{2}}\left[\ln \left(\frac{K}{S_{t}}\right)\right]^{(j-2)}\right. \\
\times\left[C_{t}^{A}(T ; K)+K e^{-r(T-t)}\right. \\
\left.\left.-S_{t} e^{-q(T-t)}\right] d K\right] .
\end{gathered}
$$

As a special case, when the American option is written on nondividend-paying asset, 
The $(T-t)$-period first-order $R N M m_{t, T-t}(1)$ is then

$$
\begin{array}{r}
m_{t, T-t}(1)=e^{r(T-t)}\left[1-\int_{S_{t}}^{\infty} \frac{1}{K^{2}} C_{t}^{A}(T ; K) d K\right] \\
-e^{r(T-t)}\left[\int _ { 0 } ^ { S _ { t } } \frac { 1 } { K ^ { 2 } } \left[C_{t}^{A}(T ; K)+K e^{-r(T-t)}\right.\right. \\
\left.\left.-S_{t}\right] d K\right]-1 .
\end{array}
$$

The $(T-t)$-period jth-order $R N M m_{t, T-t}(j)(j \geq 2)$ is then

$$
\begin{aligned}
& m_{t, T-t}(j) \\
& =j e^{r(T-t)}\left[\int_{S_{t}}^{\infty} \frac{(j-1)-\ln \left(K / S_{t}\right)}{K^{2}}\right. \\
& \left.\quad \times\left[\ln \left(\frac{K}{S_{t}}\right)\right]^{(j-2)} C_{t}^{A}(T ; K) d K\right] \\
& +j e^{r(T-t)}\left[\int_{0}^{S_{t}} \frac{(j-1)-\ln \left(K / S_{t}\right)}{K^{2}}\left[\ln \left(\frac{K}{S_{t}}\right)\right]^{(j-2)}\right. \\
& \left.\quad \times\left[C_{t}^{A}(T ; K)+K e^{-r(T-t)}-S_{t}\right] d K\right] .
\end{aligned}
$$

The condition of not exercising the calls early in Corollary 2 is moderate and easy to satisfy, especially for OTM or at-the-money (ATM) American calls, since the strike price is greater than (or equal to) the stock price and the dividend rate is not greater than the interest rate in most cases ${ }^{5}$. Practically, one can choose deeply OTM American calls because they are unlikely to be exercised before expiration ${ }^{6}$.

For the relation between $m_{t, \tau}(j)$ and $m_{t, T-t}(j)$, the following theorem holds.

Theorem 3. Under the martingale pricing measures $\pi^{*}$ and the assumption that the $\tau$-period returns are independently and identically distributed, the first four RNMs of $\log \left(R_{t, \tau}\right)$, $m_{t, \tau}(j)=E_{\pi^{*}}\left(\left[\log \left(R_{t, \tau}\right)\right]^{j}\right)(j=1,2,3,4)$ are given by

$$
\begin{gathered}
m_{t, \tau}(1)=\frac{1}{N} m_{t, T-t}(1) \\
m_{t, \tau}(2)=\frac{1}{N}\left[\left(\frac{1}{N}-1\right)\left[m_{t, T-t}(1)\right]^{2}+m_{t, T-t}(2)\right] \\
m_{t, \tau}(3)=\frac{1}{N}\left[\left(\frac{1}{N}-1\right)\left(\frac{1}{N}-2\right) m_{t, T-t}(1)\right. \\
+3\left(\frac{1}{N}-1\right) m_{t, T-t}(1) m_{t, T-t}(2) \\
\left.+m_{t, T-t}(3)\right]
\end{gathered}
$$

$$
\begin{aligned}
m_{t, \tau}(4)=\frac{1}{N}[ & \left(\frac{1}{N}-1\right)\left(\frac{1}{N}-2\right)\left(\frac{1}{N}-3\right)\left[m_{t, T-t}(1)\right]^{4} \\
& +6\left(\frac{1}{N}-1\right)\left(\frac{1}{N}-2\right) \\
& \times\left[m_{t, T-t}(1)\right]^{2} m_{t, T-t}(2) \\
& +3\left(\frac{1}{N}-1\right)\left[m_{t, T-t}(2)\right]^{2} \\
& +4\left(\frac{1}{N}-1\right) m_{t, T-t}(1) m_{t, T-t}(3) \\
& \left.+m_{t, T-t}(4)\right]
\end{aligned}
$$

where $N$ is the number of the $\tau$-period intervals from $t$ to $T$; that is, $N=(T-t) / \tau$.

Proof. The proof is given in Appendix B.

The right-hand sides of (5)-(6) show that the RNMs are the integrals of option prices over a range of strike prices $\left[0, S_{t}\right)$ and $\left[S_{t}, \infty\right)$ with two singular points 0 and $\infty$. Given a continuum of strike prices over the intervals, calculating the integrals using a numerical method is straightforward. However, only a finite number of traded options with discrete strike prices are available in a real market. Hence, we employ a more practical and effective curve-fitting method to handle the issue of option availability (see Appendix C.2 for details) and use the trapezoidal numerical method ${ }^{8}$ to numerically evaluate the integral (see Appendix C.1).

2.2. Recovering the RND within the Entropic Pricing Framework. To estimate an RND for option valuation, we use the maximum entropy framework with risk-neutral moment constraints. We initially assign equal probabilities to each possible future price (or gross return) of the underlying asset; that is, we assume the prior empirical asset returns are uniformly distributed. In the discrete case, we denote the empirical probability $\pi_{i}=1 / I, i=1,2, \ldots, I$, where $I$ is the number of return realizations. We then incorporate the constraints of RNMs recovered from options into the entropic pricing framework. The issue of estimating the riskneutral (equivalent martingale) measure $\pi^{*}$ reduces to a constrained optimization problem following the KullbackLeibler information criterion ${ }^{9}$ :

$$
\begin{aligned}
& \widehat{\pi}^{*}=\underset{\pi_{i}^{*}>0}{\arg \min } \sum_{i=1}^{I} \pi_{i}^{*} \log \left(\frac{\pi_{i}^{*}}{\pi_{i}}\right), \\
& \text { s.t. }\left\{\begin{array}{l}
\sum_{i=1}^{I} \pi_{i}^{*}\left[\log \left(R_{t-(I-i+1) \tau, \tau}\right)\right]^{j}=m_{t, \tau}(j), \\
m_{t, \tau}(0)=1, \quad j=0,1,2, \ldots, J
\end{array}\right.
\end{aligned}
$$

where $\pi_{i}^{*}$ denotes the risk-neutral (martingale) probability of the underlying asset's gross return from time $t-(I-i+1) \tau$ to $t-(I-i) \tau$ and $R_{t-(I-i+1) \tau, \tau}$ and $m_{t, \tau}(j)$ are the RNMs serving 
as constraints ${ }^{10}$. This valuation framework with RNM constraints subsumes the typical canonical valuation approach (e.g., Stutzer [2] and Liu [4]). The optimal solution $\hat{\pi}_{i}^{*}$ is given as

$$
\hat{\pi}_{i}^{*}=\frac{\exp \left(\sum_{j=1}^{J} \lambda_{j}^{*}\left[\log \left(R_{t-(I-i+1) \tau, \tau}\right)\right]^{j}\right)}{\sum_{i=1}^{I} \exp \left(\sum_{j=1}^{J} \lambda_{j}^{*}\left[\log \left(R_{t-(I-i+1) \tau, \tau}\right)\right]^{j}\right)},
$$

where the Lagrange multiplier vector $\lambda^{*}=\left(\lambda_{1}^{*}, \lambda_{2}^{*}, \ldots, \lambda_{J}^{*}\right)$ is obtained by solving the following convex optimization problem ${ }^{11}$ :

$$
\begin{aligned}
& \lambda^{*}=\underset{\lambda_{1}, \lambda 2, \ldots, \lambda_{J}}{\arg } \min \sum_{i=1}^{I} \exp \left(\sum _ { j = 1 } ^ { J } \lambda _ { j } \left[\left(\log R_{t-(I-i+1) \tau, \tau}\right)^{j}\right.\right. \\
& \left.\left.-m_{t, \tau}(j)\right]\right)
\end{aligned}
$$

2.3. Risk-Neutral Price Paths and Optimal Exercise Strategy. With the risk-neutral distribution $\hat{\pi}^{*}=\left(\widehat{\pi}_{1}^{*}, \hat{\pi}_{2}^{*}, \ldots, \widehat{\pi}_{I}^{*}\right)$ given in (12), the independent random sample of future gross returns can be drawn from the set of historical gross returns. Risk-neutral price paths for the underlying asset are then generated. Specifically, starting with time $t$, each historical return $R_{t-(I-i+1) \tau, \tau}$ is associated with a risk-neutral probability $\hat{\pi}_{i}^{*}(i=1,2, \ldots, I)$. Then a sample of $N$ returns $\left(\widetilde{R}_{t, \tau}, \widetilde{R}_{t+\tau, \tau}, \ldots, \widetilde{R}_{t+(N-1) \tau, \tau}\right)$, where $N=(T-t) / \tau$, as previously defined, is randomly drawn from the above riskneutral distribution employing the inverse transform method (Brandmiarte [10, pp. 230-232]). A risk-neutral price path is then generated. We repeat this procedure $M$ times and obtain $M$ risk-neutral price paths as

$$
\begin{aligned}
S_{t+\tau}^{(k)} & =S_{t} \widetilde{R}_{t, \tau}^{(k)}, S_{t+2 \tau}^{(k)}=S_{t} \widetilde{R}_{t, \tau}^{(k)} \widetilde{R}_{t+\tau, \tau}^{(k)}, \ldots, S_{N}^{(k)} \\
& =S_{t} \prod_{n=1}^{N} \widetilde{R}_{t+(n-1) \tau, \tau}^{(k)}, \quad(k=1,2, \ldots, M),
\end{aligned}
$$

where $\widetilde{R}_{t+(n-1) \tau, \tau}^{(k)}$ is the $n$th random sample of the gross return along the $k$ th underlying path.

Next, we determine the optimal exercise strategy for each of the risk-neutral paths. This study utilizes the LSM algorithm of Longstaff and Schwartz [5] to determine the optimal strategy for an American option over $M$ underlying price paths. Since the LSM algorithm directly uses sample paths simulated under a risk-neutral measure, averaging the payoffs of all paths yields the final payoff of the option.

The LSM algorithm consists of three steps: approximating the holding value, comparing the holding value with the immediate exercise value, and averaging the resulting payoffs of all paths (for full details of the LSM algorithm, see [5]). The crucial feature of the LSM algorithm lies in estimating the continuously holding value by a linear combination of simple basis functions at each early exercise point along each path. As recommended by Longstaff and Schwartz [5] and
Stentoft [11] $]^{12}$, the set of Legendre polynomial basis functions $\left\{1,2\left(S_{t_{n}} / K\right)-1,6\left(S_{t_{n}} / K\right)^{2}-6\left(S_{t_{n}} / K\right)+1\right\}$ is adopted to implement the LSM algorithm in this paper, where $S_{t_{n}}$ is the underlying asset price at potential exercise time $t_{n}$, where $t_{n}=t+n \tau$ for $n=1,2, \ldots, N$.

2.4. Option Pricing Algorithm. With the optimal exercise strategy for each of the $M$ underlying paths, an American call or put option expiring at time $T$ with a strike price $K$ can be valued as

$$
\begin{aligned}
& \text { For a Call, } C_{t}^{A}(T ; K) \\
& \qquad=\frac{1}{M} \sum_{k=1}^{M} e^{-r\left(t_{n}^{(k)}-t\right)} \max \left(0, S_{t} \prod_{n=1}^{n^{(k)}} \widetilde{R}_{t+(n-1) \tau, \tau}^{(k)}-K\right),
\end{aligned}
$$

For a Put, $P_{t}^{A}(T ; K)$

$$
=\frac{1}{M} \sum_{k=1}^{M} e^{-r\left(t_{n}^{(k)}-t\right)} \max \left(0, K-S_{t} \prod_{n=1}^{n^{(k)}} \widetilde{R}_{t+(n-1) \tau, \tau}^{(k)}\right),
$$

where $t_{n}^{(k)}=t+n^{(k)} \tau$ is the optimal exercise time of sample path $k$ based on the LSM algorithm. Note that, as described above, the option price is given as the average of the discounted payoff because the set of drawn samples is already risk-neutral.

\section{Simulation Testing}

In this section, we test the pricing accuracy of our RMEL method based on simulated data by benchmarking against the methods of Black-Scholes for pricing American calls ${ }^{13}$ and Crank-Nicolson finite difference (FD) for pricing American puts. We also investigate the pricing errors of our method and that of Liu [4] (Liu10) ${ }^{14}$. We simulate a sample of daily asset returns from a geometric Brownian motion (GBM) as well as a sample of American call and put options on this asset. With these two samples, we analyze the pricing accuracy with more details specified in the following subsections.

As a proof of concept, for the American calls, the RMEL approach should give the correct results as the Black-Scholes formula does when the underlying price process is modeled by GBM. For the American puts, the prices from RMEL should also be quite close to those from the method of FD. In addition, the RMEL method should work independently of the underlying growth rate in the GBM model.

3.1. Initial Setting. For ease of comparison, the parameters from Liu [4] are used in this simulation as follows.

(i) Valuation date $t$ : January 1, 2007

(ii) Expiration date T: January 1, 2008

(iii) Strike price $K$ : 40 
(iv) Risk-free interest rate $r$ : 6\%

(v) Volatility $\sigma: 40 \%$

(vi) Dividend yield q: 0\%.

3.2. Samples of Returns and Options. We simulate a sample of gross returns in which the underlying asset price $S_{t}$ is assumed to follow a GBM:

$$
d S_{t}=(\mu-q) S_{t} d t+\sigma S_{t} d \omega_{t}
$$

where $\mu$ is the drift term and $\omega_{t}$ is the standard Wiener process. Under this assumption, the continuously compounded $\tau$-year gross return is log-normally distributed and given by

$$
R_{t, \tau}=\exp \left(\left(\mu-q-\frac{\sigma^{2}}{2}\right) \tau+\sigma \sqrt{\tau} \epsilon\right),
$$

where $\epsilon$ is standard normal.

According to (17) and with the parameter values above, we generate 365 daily gross returns ${ }^{15}$ for both the risk-neutral case $(\mu=r=6 \%)$ and the unrealistic case of a growth of $\mu=$ $100 \%$ and treat them as historical returns of $R_{t-(I-i+1) \tau, \tau}(i=$ $1,2, \ldots, I ; I=365 ; \tau=1$ day), as previously defined in Section 2.2.

For the purpose of option valuation, we need a sample of American call and put options for this simulation experiment. To enable comparison, the same options as those in Liul0 with different underlying prices are used here. The value of underlying price $S_{t}$ varies from 36 to 44 with a 2-point increment (i.e., 36, 38, 40, 42, and 44) and other values of $K, r, \sigma, t, T$, and $q$ are fixed as those given above. Given that $q=0$, it is never optimal to exercise an American call early on a nondividend-paying underlying asset. Hence, the value of the above American call options can be calculated using the Black-Scholes formula. For the American puts, we calculate their values using the widelyused Crank-Nicolson FD method using an $800 \times 800$ grid. The sample of call and put options is treated as the traded options and their values are considered to be the "true" market prices.

In addition to the above options to be priced, we also need to generate a sample of call options in order to estimate the RNMs. Apparently this sample is different from the sample of calls used above for the valuation purpose. In the simulation experiment, we need to estimate a set of RNMs for each of the options to be valued. Even though the theoretical values of $m_{t, T-t}(j)$ are independent of $S_{t}$ (see, (19)), their estimates using option data are a function of $S_{t}$ (see (5)-(8)). Recall that for the valuation purpose, we take five calls and five puts with different asset prices in the experiment. When the asset prices are different, the estimates of the RNMs may not be exactly the same. Accordingly, we generate eight samples of call options to estimate the RNMs and then the RND for each of the given asset prices. Hence, the options with the given asset price are valued based on their corresponding RND. In each of eight samples for a given asset price, there are four call options being in the money (ITM), that is, their strike prices being lower than the asset price, and four being out of the money (OTM), that is, their strike prices higher. More specifically, the strike prices for asset price $36,38,40,42$, and 44 are $(16,22,28,34,40,46,52,58),(18,24,30,36,42,48,54$, $60),(20,26,32,38,44,50,56,62),(22,28,34,40,46,52,58$, $64)$, and $(24,30,36,42,48,54,60,66)$, respectively.

3.3. Estimation of RNMs and RNDs. The mathematical form of the risk-neutral probability distribution $\hat{\pi}_{i}^{*}$ in (12) depends on the number $J$ of the RNM constraints $m_{t, \tau}(j)(j=$ $0,1,2, \ldots, J)$. For example, if $J$ is zero, no constraint is imposed and the distribution $\hat{\pi}_{i}^{*}$ is uniform. Under the assumption of GBM, $\hat{\pi}_{i}^{*}$ is normally distributed and can be exactly characterized by its first and second moments. Hence, we use the first two moments as constraints for deriving the RND and only estimate $m_{t, \tau}(1)$ and $m_{t, \tau}(2)$.

We first use the generated 8 call options specified in Section 3.2 to calculate $m_{t, T-t}(1)$ and $m_{t, T-t}(2)$ based on Corollary 2 and then convert them to $m_{t, \tau}(1)$ and $m_{t, \tau}(2)$ according to Theorem 3 as discussed in Section 2.1. Two issues involved in the integrals in Corollary 2 need to be addressed. For any traded option with a specified maturity in the real market, the number of available strike prices (or option prices) is finite. The first issue therefore concerns the limited availability of strike prices (or option prices). To solve this problem, we use a curve-fitting method to generate "implied" options with a range of strike prices. We first calculate implied volatilities using the Black-Scholes formula based on the simulated (or market-available) call options. These implied volatilities are then used to form a fitted function of the volatility surface via the cubic splines method. Given the fitted function of the volatility surface, we can find the required volatilities and plug them into the Black-Scholes formula to obtain the "implied" call option prices. The details are in Appendix C.2. The second issue is the calculation of the integrals on the right hand side of (5) and (6). A numerical integration is carried out using the trapezoidal method, with the integration intervals split into $m=80$ equal subintervals. The technical details of calculating the RNMs are given in Appendix C.1 and also outlined in footnotes 7 and 8 .

Through the procedure specified above, we use only 8 options that are usually available in a real market ${ }^{16}$ to obtain the RNMs $\left(m_{t, \tau}(1)\right.$ and $\left.m_{t, \tau}(2)\right)$. We can calculate the true (theoretical) values for the first two moments in a riskneutral world based on the above GBM. As is well-known, the solution for the above GBM in a risk-neutral world is

$$
\log \left(R_{t, \tau}\right)=\left(r-q-\frac{\sigma^{2}}{2}\right) \tau+\sigma \sqrt{\tau} \epsilon
$$

where $\epsilon$ is a standard normal random variable. Then the theoretical values, denoted as $m_{t, T-t}(j)^{\text {true }}=E\left(\left[\log \left(R_{t, T-t}\right)\right]^{j}\right)$ for the $(T-t)$-period return and $m_{t, \tau}(j)^{\text {true }}=E\left(\left[\log \left(R_{t, \tau}\right)\right]^{j}\right)$ 
for the $\tau$-period return $(j=1,2)$, can be easily calculated as follows:

$$
\begin{gathered}
m_{t, T-t}(1)^{\text {true }}=\left(r-q-\frac{\sigma^{2}}{2}\right)(T-t), \\
m_{t, \tau}(1)^{\text {true }}=\left(r-q-\frac{\sigma^{2}}{2}\right) \tau, \\
m_{t, T-t}(2)^{\text {true }}=\left[\left(r-q-\frac{\sigma^{2}}{2}\right)(T-t)\right]^{2}+\sigma^{2}(T-t), \\
m_{t, \tau}(2)^{\text {true }}=\left[\left(r-q-\frac{\sigma^{2}}{2}\right) \tau\right]^{2}+\sigma^{2} \tau .
\end{gathered}
$$

Since there are multiple underlying asset prices in the experiment, we extract the RNMs for each underlying price. Table 1 reports the estimates of RNMs and their theoretical values.

Table 1 shows that the RNM estimates are nearly the same as their theoretical values. This demonstrates that eight options can effectively capture the shape of distribution of the underlying asset returns. Furthermore, the estimated RNMs are almost indistinguishable for both moments, even though the underlying prices are different. This indicates that the two moments obtained are exactly "risk-neutral" and are not related to the current asset price. It can be seen again from (19) that the RNMs are only determined by interest rate and volatility and not by the underlying asset price.

In addition to the estimated RNMs, we also need to simulate return series following (17) for recovering the RND according to (12) and (13). In this experiment, we simulate two series of 365 returns; that is, $R_{t-(365-i+1) \tau, \tau}(i=1,2, \ldots, 365)$ for growth rates of $\mu=6 \%$ and $100 \%$, respectively, and other parameters at values of $\sigma=40 \%, T-t=1$, and $\tau=1 / 365$. Thus, two corresponding RNDs of $\hat{\pi}^{*(1)}=$ $\left(\widehat{\pi}_{1}^{*(1)}, \widehat{\pi}_{2}^{*(1)}, \ldots, \widehat{\pi}_{365}^{*(1)}\right)$ and $\hat{\pi}^{*(2)}=\left(\widehat{\pi}_{1}^{*(2)}, \widehat{\pi}_{2}^{*(2)}, \ldots, \widehat{\pi}_{365}^{*(2)}\right)$ are recovered.

3.4. Pricing Results and Comparison Analysis. This section first presents the pricing results and then compares them with the "true" values. Tables 2 and 3 report the estimated prices of American calls and puts using the RMEL method and the method of Liul0.

Tables 2 and 3 report that the estimated prices of the RMEL method are fairly close to the "true" prices for both growth rates across a range of asset prices, especially for put options. The absolute differences between the RMEL and the Black-Scholes formula are all below 1\%; see columns four and six in Table 2 . Even below $0.32 \%$ for put options, see columns four and six in Table 3. It appears that the RMEL method is comparable to the Black-Scholes formula for American calls and to the FD method for American puts. Furthermore, for each price estimate in both growth rates of $6 \%$ and $100 \%$, two pricing errors are so small that the difference between two estimates is slight. This finding again illustrates that the RMEL method is actually independent of the growth rate.

Taking the absolute difference between the estimated value from a method and the "true" value as a measure for judging price deviation, the absolute difference from our method is a little bit higher than that from Liul0 for both call and put options when the asset price is 42 , as well as for call options with the $6 \%$ growth rate when the asset price is 38 , but the difference is still slight since the absolute differences are so small. Fortunately and importantly, all the other absolute differences from RMEL are lower and even much smaller than those from Liul0. This suggests that the magnitudes of the pricing error resulting from RMEL method are, overall, smaller than those from Liul0.

In brief, these results indicate that both American calls and puts can be priced rather accurately by our RMEL approach in the simulated market, and this method provides better precision than the method of Liul0. It should also be noted that, there is no discernible relation between the accuracy of pricing and moneyness ${ }^{17}$.

\section{Comparison Based on IBM Option Data}

4.1. Data Descriptions. We collected daily data of IBM call and put options from the website http://finance.yahoo.com/. The data cover July 31, 2008 through January 30, 2009 for a total of 127 trading days. After filtering ${ }^{18}, 4430$ calls and 4430 put options remain with the time to maturity being 16 to 357 days. The closing price of IBM stock is treated as the underlying price and here the discrete dividends (paid quarterly) are taken into consideration. Depending on the valuation date, the dividend payment dates are assumed to be on August 6, 2008 and November 6, 2008, and the corresponding quarterly dividends are actually $\$ 0.5$ according to the downloaded data. Table 4 briefly describes the filtered data of IBM calls and puts.

The daily US Treasury yield curve rate from one month to 30 years for each valuation date is used or interpolated linearly for any particular time to maturity as the corresponding continuous, constant risk-free interest rate. The yield curve is obtained directly from the website of the US Department of the Treasury, http://www.treasury.gov/resource-center/datachart-center/interest-rates/Pages/default.aspx.

4.2. Valuation Methods. The empirical results for our method indicate that the third and fourth moments are highly linearly dependent of the first two moments. This implies that the third and fourth moments can be expressed as a linear combination of the first two moments. The linear dependence could result in an ill-conditioned problem when solving the optimization problem, that is, (11). Mathematically, if the matrix $\left[\left(\log \left(R_{t-(I-i+1) \tau, \tau}\right)^{j}\right)\right]_{J \times I}$ is not a full rank, the Lagrange multipliers cannot be uniquely determined (Agmon et al. [7]). Consequently, many risk-neutral probabilities equal zero. This problem actually appears many times while pricing IBM options. Here is an example of options with trading date of July 31, 2008, stock price of $\$ 127.98$, and time to maturity of 78 days. In the case of three moment constraints, 176 out of 260 calculated risk-neutral probabilities are nonzero; in the case of four moment constraints, only 74 among 260 are nonzero, whereas all the probabilities are positive when using the first two moment constraints. Hence, in our 
TABLE 1: Recovered moments and their theoretical values for a range of initial underlying prices $\left(S_{t}=36,38,40,42,44\right)$ in the simulation.

\begin{tabular}{lccccc}
\hline Underlying price & 36 & 38 & 40 & 42 & 44 \\
\hline 1st order moment & -0.0200 & -0.0200 & -0.0200 & -0.0200 & -0.0200 \\
$\left(m_{t, T-t}(1)\right)$ & -0.0200 & -0.0200 & -0.0200 & -0.0200 & -0.0200 \\
\hline 2nd order moment & 0.1605 & 0.1605 & 0.1605 & 0.1604 \\
$\left(m_{t, T-t}(2)\right)$ & 0.1604 & 0.1604 & 0.1604 & 0.1604 & 0.1604 \\
\hline
\end{tabular}

Note: the first two moment estimates for $\log \left(R_{t, T-t}\right)$ with each of the underlying prices $\left(S_{t}\right)$ are compared to their corresponding theoretical values under the GBM, calculated by (19) with the parameters $r=0.06, \sigma=40 \%, q=0, T-t=1$.

These moments are recovered using only 8 call options discussed in Section 3.2. For both moments, the top row reports the estimated values, and the bottom row reports the theoretical (true) values.

TABLE 2: Averaged prices of American call options for a range of asset prices $\left(S_{t}=36,38,40,42,44 ; K=40\right)$.

\begin{tabular}{|c|c|c|c|c|c|c|}
\hline Method $\downarrow$ & Asset price $S_{t}$ & $\begin{array}{l}\text { Black-Scholes } \\
\text { formula } C_{t}\end{array}$ & $\begin{array}{c}\text { Growth rate } \\
\mu=6 \%\end{array}$ & $\begin{array}{c}\text { Difference (\%) } \\
\frac{\text { Estimated value }-C_{t}}{C_{t}}\end{array}$ & $\begin{array}{l}\text { Growth rate } \\
\mu=100 \%\end{array}$ & $\begin{array}{c}\text { Difference }(\%) \\
\text { Estimated value }-C_{t} \\
C_{t}\end{array}$ \\
\hline \multirow{5}{*}{ RMEL } & 36 & 5.041 & 5.013 & -0.555 & 5.029 & -0.238 \\
\hline & 38 & 6.164 & 6.123 & -0.665 & 6.151 & -0.211 \\
\hline & 40 & 7.389 & 7.357 & -0.433 & 7.376 & -0.176 \\
\hline & 42 & 8.708 & 8.653 & -0.632 & 8.679 & -0.333 \\
\hline & 44 & 10.112 & 10.065 & -0.465 & 10.092 & -0.198 \\
\hline \multirow{5}{*}{ Liul0 } & 36 & 5.041 & 5.076 & 0.694 & 5.074 & 0.655 \\
\hline & 38 & 6.164 & 6.186 & 0.357 & 6.201 & 0.600 \\
\hline & 40 & 7.389 & 7.426 & 0.501 & 7.458 & 0.934 \\
\hline & 42 & 8.708 & 8.730 & 0.253 & 8.723 & 0.172 \\
\hline & 44 & 10.112 & 10.169 & 0.564 & 10.158 & 0.455 \\
\hline
\end{tabular}

Note: the numbers in the first two columns represent, respectively, asset prices and the corresponding true Black-Scholes prices (as the underlying asset pays no dividend). Columns 3 and 5 report the price estimates with the growth rates of $6 \%$ and $100 \%$ for the two methods, and each reported value represents an estimate for a particular combination of growth rate and asset price. The values reported in columns 4 and 6 are the corresponding difference between the estimated and the "true" Black-Scholes prices, respectively. The difference is calculated by dividing the estimated price minus the Black-Scholes price by the Black-Scholes price. For both RMEL and Liu10, each reported price estimate is the average of the prices over three independent simulations. In each simulation, 100,000 risk-neutral price paths are generated and each path is divided into $P=73$ exercise opportunities.

TABLE 3: Averaged prices of American puts for a range of asset prices $\left(S_{t}=36,38,40,42,44 ; K=40\right)$.

\begin{tabular}{|c|c|c|c|c|c|c|}
\hline Method $\downarrow$ & Asset price $S_{t}$ & $\begin{array}{l}\text { Crank-Nicolson } \\
\text { FD Formula } P_{t}\end{array}$ & $\begin{array}{c}\text { Growth rate } \\
\mu=6 \%\end{array}$ & $\begin{array}{c}\begin{array}{c}\text { Difference }(\%) \\
\text { Estimatedvalue }-P_{t}\end{array} \\
P_{t}\end{array}$ & $\begin{array}{c}\text { Growth rate } \\
\mu=100 \%\end{array}$ & $\begin{array}{c}\begin{array}{c}\text { Difference (\%) } \\
\text { Estimatedvalue }-P_{t}\end{array} \\
P_{t}\end{array}$ \\
\hline \multirow{5}{*}{ RMEL } & 36 & 7.109 & 7.094 & -0.211 & 7.091 & -0.253 \\
\hline & 38 & 6.154 & 6.139 & -0.244 & 6.145 & -0.146 \\
\hline & 40 & 5.318 & 5.305 & -0.244 & 5.301 & -0.320 \\
\hline & 42 & 4.588 & 4.575 & -0.283 & 4.575 & -0.283 \\
\hline & 44 & 3.953 & 3.941 & -0.304 & 3.945 & -0.202 \\
\hline \multirow{5}{*}{ Liul0 } & 36 & 7.109 & 7.145 & 0.506 & 7.138 & 0.407 \\
\hline & 38 & 6.154 & 6.195 & 0.666 & 6.167 & 0.211 \\
\hline & 40 & 5.318 & 5.364 & 0.865 & 5.360 & 0.789 \\
\hline & 42 & 4.588 & 4.596 & 0.174 & 4.598 & 0.217 \\
\hline & 44 & 3.953 & 3.992 & 0.987 & 3.980 & 0.683 \\
\hline
\end{tabular}

Note: the reported numbers in the first two columns represent, respectively, the asset prices and the corresponding "true" prices calculated using the CrankNicolson finite difference $(800 \times 800 \mathrm{Grid})(r=0.06)$. Columns 3 and 5 report the price estimates with the growth rates of $6 \%$ and $100 \%$ for the two methods. Each reported value represents an estimate of option price for a particular combination of growth rate and asset price. The values reported in Columns 4 and 6 are the corresponding difference between the estimated price and the Crank-Nicolson finite difference prices, respectively. The difference is calculated by dividing the estimated price minus the "true" price of the Crank-Nicolson finite difference by the Crank-Nicolson finite difference price. For both RMEL and Liu10, each price estimate is the average of the values over three independent simulations. Each of the simulations generates 100,000 sample price paths and each path is divided into 73 potential exercise opportunities. 
TABLE 4: Data description of IBM calls and puts.

\begin{tabular}{|c|c|c|c|c|c|}
\hline & & Mean & Standard deviation & Minimum & Maximum \\
\hline \multirow{4}{*}{ Call } & Number of options & 4430 & & & \\
\hline & Market prices & 13.653 & 14.245 & 0.075 & 69.550 \\
\hline & Moneyness $S_{t} / K$ & 1.077 & 0.329 & 0.514 & 2.292 \\
\hline & Expiration & 119.008 & 71.886 & 16 & 357 \\
\hline \multirow{4}{*}{ Put } & Number of options & 4430 & & & \\
\hline & Market prices & 13.614 & 15.188 & 0.075 & 75.200 \\
\hline & Moneyness $K / S_{t}$ & 1.010 & 0.283 & 0.436 & 1.946 \\
\hline & Expiration & 119.008 & 71.886 & 16 & 357 \\
\hline
\end{tabular}

Note: the call and put prices here are the midvalues of the bid-ask quotations. Moneyness is defined as the IBM stock closing price divided by the strike price, $S_{t} / K$, for call options and the strike price divided by the closing price, $K / S_{t}$, for put options. The time to expiration is measured in days.

empirical investigation, only the first two moment constraints are incorporated into our pricing framework to price IBM options.

The empirical investigation involves four pricing methods. For IBM call options, our RMEL method with two moment constraints, the method of Liul0 without moment constraint, and Alcock and Auerswald [6] (AA10) with option-constraint, and, for IBM puts, the Crank-Nicolson FD (FD). It should be pointed out here that due to the fact that the IBM stock pays discrete dividends, the factor $e^{-q(T-t)}$ in (3)-(4) would be replaced with $\left(1-D / S_{t}\right)$ when calculating the RNMs, that is, (7)-(8), where $D$ is the present value of dividend payments at time $t$.

For each reported price, three independent Monte Carlo runs are carried out and the resultant prices are averaged as option prices. Each simulation generates 10,000 risk-neutral price paths, with each path made up of one-day gross returns sampled from the risk-neutral measure. The simulated riskneutral stock price for every path on the exdividend date is reduced exactly by the dividend amount, and the previous 260 daily closing prices are used to calculate the historical gross returns. Each path is further divided into a number of potential exercise steps according to the following rule. If the number of days to expiration is less than 50 , the size of the step is set to one day; otherwise, the number of days in one step is an integer part of the number of days to expiration divided by 50 , while the last step can cover fewer days than the remaining ones. The least-squares algorithm uses the first two terms of the Legendre polynomial plus a constant term, $\left\{1,2\left(S_{t} / K\right)-1,6\left(S_{t} / K\right)^{2}-6\left(S_{t} / K\right)+1\right\}$, as basis functions. For integral calculations, each of the integration intervals $\left[S_{t}, K_{\max }\right],\left[K_{\max }, K_{\infty}\right],\left[K, K_{\min }\right]$, and $\left[K_{\min }, S_{t}\right]$ is split into $m=80$ nonoverlapping intervals of equal length.

With regard to the AA10 method, we use the same criteria as those in AA10 to choose an option as the constraint. For pricing a call option, a prior day observed call option with the same strike price and the same expiration is taken, when pricing a put, the preferred constraint is a prior day observed call option with the same expiration date and moneyness closest to 1.0, and if no adequate option can be found on the day prior, the $i$ th call option with trading date of $t_{i}$ and maturity of $T_{i}$ traded in the previous five days with time to maturity equal to $\max _{T_{j}-t_{j} \leq T-t}\left(T_{i}-t_{i}\right)$ and moneyness closest to 1.0 is chosen as the constraint.

Finally, for the FD method, since the stock grid is fixed in the backward induction of finite difference, the option value on the exdividend date is adjusted downward by using quadratic interpolation to reflect the effect of dividend payments and thus corresponds to the option value for the dividend-adjusted stock price. If the dividend-adjusted stock price is less than the second lowest stock price on the grid, however, the corresponding option value is set to the option value for the lowest stock price in the grid and no interpolation is carried out. In addition, volatility is calculated as the standard deviations of the daily returns multiplied by square root of 252, based on the previous 260 daily IBM stock closing prices. A default grid of one-day time step and a grid of stock price spacing of $\Delta(\ln S)=\sigma \sqrt{3 \tau}$ as suggested in Hull $[12$, pp.443] are used.

4.3. Empirical Results. The valuation results are summarized and compared using 12 categories of moneyness and time to expiration. Four levels of moneyness are given as: less than 0.85 (i.e, DOTM), from 0.85 to 1.00 (i.e., OTM), from 1.00 to 1.15 (i.e., in-the-money, ITM), and greater than 1.15 (i.e., DITM). There are three groups of time to expiration: from 16 to 60 days (short term), from 61 to 160 days (medium term), and from 161 to 357 days (long term). In addition, the following frequently-used loss measures are used to analyze the pricing errors: the mean percentage error (MPE), the mean square error (MSE), and the mean absolute percentage error (MAPE) ${ }^{19}$.

Tables 5 and 6 summarize the pricing results from different valuation methods corresponding to either call or put options, and the number of options is also shown for each category.

Several observations of pricing errors between IBM calls and puts using RMEL method can be made. First, the RMEL pricing error is distributed more evenly for calls; for example, the MAPE is around $15 \%$ to $20 \%$ over 12 categories for calls, while the MAPE of puts generally decreases dramatically with moneyness. Table 6 shows that the MAPE of RMEL is much less than $10 \%$ for the ITM and DITM cases but basically 
TABLE 5: MPE, MSE, and MAPE of IBM call price estimates compared to IBM market prices for a range of moneyness and time to expiration.

\begin{tabular}{|c|c|c|c|c|c|c|c|c|c|c|}
\hline \multirow{2}{*}{ Moneyness $\left(S_{t} / K\right)$} & \multirow{2}{*}{$\begin{array}{c}\text { Expiration } \\
\text { Method }\end{array}$} & \multicolumn{3}{|c|}{$16-60$ (short) } & \multicolumn{3}{|c|}{ 61-160 (medium) } & \multicolumn{3}{|c|}{ 161-357 (long) } \\
\hline & & RMEL & Liul0 & AA10 & RMEL & Liul0 & AA10 & RMEL & Liul0 & AA10 \\
\hline \multirow{4}{*}{$\begin{array}{l}S_{t} / K<0.85 \\
(\mathrm{DOTM})\end{array}$} & Count & & 199 & & & 480 & & & 529 & \\
\hline & MPE (\%) & -5.352 & -14.208 & 6.214 & -3.508 & -3.931 & -7.035 & -6.035 & -7.932 & -12.136 \\
\hline & MSE & 1.823 & 5.636 & 1.402 & 1.489 & 4.785 & 2.902 & 1.806 & 4.757 & 6.238 \\
\hline & MAPE (\%) & 14.735 & 26.291 & 21.035 & 15.225 & 35.138 & 20.186 & 15.132 & 32.248 & 17.301 \\
\hline \multirow{4}{*}{$\begin{array}{l}S_{t} / K \in[0.85,1.00) \\
(\mathrm{OTM})\end{array}$} & Count & & 371 & & & 304 & & & 290 & \\
\hline & MPE (\%) & -5.130 & 2.620 & 5.326 & -4.919 & -4.634 & -18.325 & -5.726 & -4.482 & -20.648 \\
\hline & MSE & 1.290 & 4.064 & 1.824 & 1.164 & 4.060 & 3.028 & 1.357 & 4.814 & 4.039 \\
\hline & MAPE (\%) & 19.868 & 44.545 & 23.894 & 16.825 & 35.937 & 27.682 & 19.781 & 42.443 & 26.026 \\
\hline \multirow{4}{*}{$\begin{array}{l}S_{t} / K \in[1.00,1.15) \\
(\mathrm{ITM})\end{array}$} & Count & & 289 & & & 248 & & & 202 & \\
\hline & MPE (\%) & -3.044 & -2.307 & -6.563 & -4.688 & -2.794 & -19.542 & -0.831 & 1.652 & -23.249 \\
\hline & MSE & 1.314 & 3.906 & 3.358 & 0.873 & 3.046 & 8.065 & 1.106 & 3.495 & 16.548 \\
\hline & MAPE (\%) & 17.060 & 34.599 & 18.175 & 18.988 & 38.985 & 25.338 & 20.091 & 41.227 & 28.065 \\
\hline \multirow{4}{*}{$\begin{array}{l}S_{t} / K \geq 1.15 \\
(\mathrm{DITM})\end{array}$} & Count & & 434 & & & 670 & & & 414 & \\
\hline & MPE (\%) & -2.181 & -3.637 & -4.212 & -2.846 & -7.860 & -9.542 & -5.096 & -8.887 & -11.254 \\
\hline & MSE & 1.386 & 4.290 & 2.921 & 1.189 & 3.915 & 8.621 & 0.725 & 3.085 & 15.245 \\
\hline & MAPE (\%) & 15.663 & 37.031 & 13.984 & 15.690 & 31.904 & 15.938 & 18.616 & 38.707 & 19.351 \\
\hline
\end{tabular}

Note: each cell represents a particular combination of moneyness and time to expiration. Three independent simulations are carried out, while 10,000 underlying price paths are generated in each simulation. The first row reports the number of call options with the corresponding combination of moneyness and time to expiration. The remaining rows show the statistic results of the MPE, RMSE, and MAPE for each combination. The total number of options analysed is 4430 , the lowest moneyness is $79.66 / 155=0.514$, the highest moneyness is $91.66 / 40=2.292$, and the time to expiration is in between 16 and 357 days.

TABLE 6: MPE, MSE, and MAPE of IBM put price estimates compared to IBM market prices for a range of moneyness and time to expiration.

\begin{tabular}{|c|c|c|c|c|c|c|c|c|c|c|c|c|c|}
\hline \multirow{2}{*}{ Moneyness $\left(K / S_{t}\right)$} & \multirow{2}{*}{$\begin{array}{l}\text { Expiration } \\
\text { Method }\end{array}$} & \multicolumn{4}{|c|}{$16-60$ (short) } & \multicolumn{4}{|c|}{ 61-160 (medium) } & \multicolumn{4}{|c|}{ 161-357 (long) } \\
\hline & & RMEL & Liul0 & AA10 & FD & RMEL & Liul0 & AA10 & FD & RMEL & Liul0 & AA10 & FD \\
\hline \multirow{4}{*}{$\begin{array}{l}K / S_{t}<0.85 \\
(\mathrm{DOTM})\end{array}$} & Count & \multicolumn{4}{|c|}{372} & \multicolumn{4}{|c|}{618} & \multicolumn{4}{|c|}{374} \\
\hline & MPE (\%) & -46.223 & -78.924 & -82.536 & -88.694 & -30.291 & -53.189 & -51.623 & -89.083 & -4.054 & 0.899 & -40.284 & -75.498 \\
\hline & MSE & 0.057 & 0.439 & 1.206 & 0.634 & 0.115 & 0.754 & 2.064 & 1.945 & 0.826 & 2.397 & 2.203 & 3.219 \\
\hline & MAPE (\%) & 49.451 & 79.437 & 82.735 & 96.226 & 36.683 & 60.518 & 59.137 & 89.083 & 24.695 & 47.858 & 45.735 & 75.498 \\
\hline \multirow{4}{*}{$\begin{array}{l}K / S_{t} \in[0.85,1.00) \\
(\mathrm{OTM})\end{array}$} & Count & \multicolumn{4}{|c|}{351} & \multicolumn{4}{|c|}{299} & \multicolumn{4}{|c|}{241} \\
\hline & MPE (\%) & -2.021 & -15.641 & -21.253 & -28.855 & -0.688 & 4.292 & -5.382 & -28.834 & -0.183 & 9.063 & -8.15 & -24.484 \\
\hline & MSE & 0.127 & 1.150 & 1.851 & 2.526 & 0.521 & 2.712 & 2.685 & 4.631 & 5.193 & 11.681 & 5.346 & 5.218 \\
\hline & MAPE (\%) & 10.390 & 37.253 & 37.564 & 48.761 & 11.254 & 33.690 & 30.258 & 31.554 & 24.518 & 46.614 & 25.458 & 24.711 \\
\hline \multirow{4}{*}{$\begin{array}{l}K / S_{t} \in[1.00,1.15) \\
(\mathrm{ITM})\end{array}$} & Count & \multicolumn{4}{|c|}{331} & \multicolumn{4}{|c|}{262} & \multicolumn{4}{|c|}{254} \\
\hline & MPE (\%) & 0.389 & 0.817 & -3.358 & 9.457 & -0.960 & -4.337 & -1.861 & -1.856 & -7.996 & -9.825 & -7.658 & -4.303 \\
\hline & MSE & 0.202 & 0.405 & 1.513 & 3.625 & 0.328 & 1.928 & 2.358 & 3.182 & 2.748 & 7.835 & 5.865 & 3.856 \\
\hline & MAPE (\%) & 3.336 & 5.780 & 5.632 & 17.023 & 3.470 & 9.756 & 5.861 & 12.354 & 9.309 & 18.275 & 10.843 & 10.473 \\
\hline \multirow{4}{*}{$\begin{array}{l}K / S_{t} \geq 1.15 \\
(\mathrm{DITM})\end{array}$} & Count & \multicolumn{3}{|c|}{239} & & \multicolumn{4}{|c|}{523} & \multicolumn{4}{|c|}{566} \\
\hline & MPE (\%) & -0.566 & -0.571 & -0.865 & 5.747 & -0.662 & -1.706 & -0.952 & 2.997 & -2.372 & -3.535 & -2.536 & 2.041 \\
\hline & MSE & 0.226 & 0.172 & 1.263 & 2.547 & 0.489 & 0.727 & 1.213 & 1.924 & 1.118 & 2.098 & 2.253 & 2.040 \\
\hline & MAPE (\%) & 1.589 & 1.822 & 1.832 & 5.899 & 1.845 & 2.250 & 1.925 & 3.928 & 2.623 & 3.811 & 3.531 & 3.624 \\
\hline
\end{tabular}

Note: each reported value is for a particular combination of moneyness and time to expiration. Three independent simulations are carried out, while 10,000 underlying price paths are generated in each simulation. The first row reports the number of put options with the corresponding combination of moneyness and time to expiration. The remaining rows show the statistic results of the MPE, RMSE, and MAPE for each combination. The total number of options analyzed is 4430 , the lowest moneyness is $40 / 91.66=0.436$, the highest moneyness is $155 / 79.66=1.946$, and the time to expiration is in between 16 and 357 days. 
increases with time to expiration. Second, from Tables 5 and 6 , based on the MPE, IBM calls are overall underpriced by the RMEL method as are IBM puts, with the exception of the case of ITM and short term to expiration. Third, the RMEL method overall outperforms methods of AA10.

Table 5 compares IBM calls priced by our methods RMEL, Liu10, and AA10. The method of RMEL shows a negative pricing bias in all combinations of moneyness and time to expiration, whereas Liul0 produces a positive bias when the option is OTM-short or ITM-long and AA10 also has two positive bias in the cases of DITM-short and ITMshort. Second, for each category, the RMEL method performs better than other methods by the important error measure MAPE although the MAPE for the RMEL method is a little bit large (over 10\%), for example, the MAPE from the RMEL method is nearly a half that from Liul0 in each category. Third, compared to the method AA10, using the measure MAPE, the errors from RMEL are overall smaller especially when option is OTM or DOTM. Finally, the methods of RMEL and AA10 apparently perform much better than Liul0 because the method of Liul0 has not used any other constraint except the necessary martingale constraint.

For IBM puts, in addition to two methods above, the benchmark valuation method FD here is also compared with RMEL. First, as reported in Table 6, RMEL method produces a negative bias in 12 categories, with the exception of ITMshort; AA10 exhibit all negative bias; Liul0 has 4 positive bias in the cases of DOTM-long, OTM-long, OTM-medium, and ITM-short; and FD has 4 positive bias for all DITM options and ITM-short options. It is understandable for the methods of RMEL, Liu10, and AA10 to produce negative bias in most of 12 categories since the least-squares algorithm provides lower bounds for American puts. Second, the magnitude of pricing error decreases with moneyness for all methods. Third, FD is fairly accurate and outperforms Liul0 for the ITM-long and DITM-long cases. These results are consistent with market behaviour, since the market seems to have placed a premium on OTM puts ever since the 1987 market crash. Fortunately, RMEL performs much better than FD, even in the cases of ITM-long and DITM-long. In addition, RMEL absolutely dominates Liul0 since Liul0 has no other constraint. Compared to AA10, from the measure MAPE, RMEL outperforms AA10 in all categories. Furthermore, all the MAPE errors from RMEL are below $10 \%$ for both the ITM and DITM cases, even reaching 1.589\% for DITM-short. For other cases over $10 \%$, it is understandable and acceptable if considering the reasons below, in the IBM option data, the price for DOTM puts is very low and the bid-ask spread is relatively quite large especially for DOTM-short options.

In summary, the empirical results again illustrate that the RMEL method produces much smaller pricing errors than Liul0 and overall outperforms method of AA10 for IBM call options. The RMEL method is more stable for IBM call options from the measure MPE and can effectively estimate call prices with a reasonable pricing error. For IBM puts, the magnitudes of the pricing bias from RMEL and AA10 are much lower than those from both Liu10 and FD methods and RMEL is better than AA10. The RMEL method also produces more accurate values for IBM puts, especially when the put option is ITM or DITM. Empirical analysis suggests that on the whole, RMEL outperforms Liu10, AA10, and FD. Meanwhile, it should be pointed out that the RNM constraints used in RMEL method and the option constraint in AA10 actually contain some more useful information so that both methods greatly outperform the method of Liul0.

\section{Conclusion}

This paper introduces an ideally extended RNMs-constrained entropic least-squares valuation method which improves the nonparametric valuation technique to price American options. Our RMEL approach uses the RNMs recovered from a much smaller set of option data as constraints to generate a better estimate of RND as the pricing measure and then incorporates it into tractable Monte Carlo techniques to price American options.

The RNMs in our valuation approach can be estimated using several call options, and we derive the general expression for extracting the RNMs. These RNMs play a significant role in deriving a better RND, owing to their ability for capturing market information without imposing any underlying structural assumption. Compared with other existing entropic valuation methods, this is an outstanding feature for the RMEL method.

With the extracted RNMs as constraints, we establish the RNMs-constrained entropy valuation framework and by solving this RNMs-constrained entropy problem, we obtain a discrete risk-neutral distribution as the unique pricing measure. Finally, the optimal exercise strategies are also achieved via the least-squares Monte Carlo algorithm and consequently the pricing algorithm of American options are obtained.

We evaluate the usefulness of our method and compare its performance in simulation environments in a number of ways with the method of Liu [4], who extends the canonical valuation to price American options. First, the results of extracting RNMs suggest that our moment estimates match rather well with the theoretical values in simulation experiments. Second, the estimated prices using the RMEL method are fairly close to the "true" prices for American call and put options in the cases of both growth rates. Consistent with the finding of Gray and Newman [13], all price estimates are less than the "true" prices for both call and put options. But the method of Liul0 method persistently exhibits a positive bias. Furthermore, the price bias of the RMEL method is more stable for two growth rates. By comparing the absolute difference between the estimated and "true" prices, the overall accuracy of our approach is higher than that of Liulo and particularly dominant in pricing American puts. Finally, it is not unreasonable to imagine that the RMEL method nests the method of Liu10 as special cases. We also empirically test our valuation approach and compare its performance with the methods of Liu10, AA10, and FD using the IBM options data. The results show that the pricing bias by our RMEL approach is lower than that by the method of AA10 for almost all the levels of moneyness and time to maturity, and the methods of RMEL and AA10 largely outperform the Liu10 and FD 
methods, whether for call options or put options. For IBM calls, the pricing errors of the RMEL method equal nearly half of that of Liul0 and the RMEL method outperforms AA10. With regard to IBM put option valuation, Liul0 performs much better than the FD when the time to maturity is short, whereas the FD outperforms Liu10 across moneyness with long maturity, but their results including those from AA10 are worse than that of RMEL method. Meanwhile, our method significantly dominates Liul0 and FD methods, especially for ITM and DITM; the RMEL method can price put options very well with a rather high accuracy. In brief, all the results suggest again that our approach performs well and much better than some benchmark approaches.

In summary, to generate a better estimate of the riskneutral distribution of the underlying assets for pricing American options, an entropy valuation with moment constraints that can be easily constructed using a small number of options is developed and tested in simulations and with IBM option data. We demonstrate that our method prices American options quite well and outperforms several benchmarks and nonparametric approaches. In principle, the RMEL method can be applied in any other artificial circumstances and real markets due to its ability to effectively capture information in the option market to generate a better estimate of the risk-neutral measure. Also the RMEL approach is applicable to other path-dependent options. Further work is required to investigate the relation between the number of the RNM constraints and pricing accuracy, and another direction is to address the numerical solution problem when more moment constraints are incorporated into the valuation framework.

\section{Appendices}

\section{A. Proof of Lemma 1}

Let $\left(\Omega, F_{t}, \pi^{*}\right)$ be the probability space over time interval $[t, T]$ with filtration $F_{t}$ and let $\Phi_{R_{t, T-t}}(x)=E_{\pi^{*}}\left(e^{i x \log \left(R_{t, T-t}\right)}\right)$ be the characteristic function of the underlying asset return $\log \left(R_{t, T-t}\right)$, where $\log \left(R_{t, T-t}\right) \equiv \log \left(S_{T} / S_{t}\right)$ and $S_{t}$ is known at time $t ; i$ is the imaginary unit under the martingale measure $\pi^{*}$. We begin with deriving the analytic form of $\Phi_{R_{t, T-t}}(x)$.

Defining a twice-continuously differentiable function with respect to $S_{T}$,

$$
f\left(S_{T}\right)=e^{i x \log \left(S_{T} / S_{t}\right)} .
$$

By the second-order version of Taylor's Theorem with integral remainder (e.g., Dudley [14, pp.522]), we have

$$
\begin{aligned}
f\left(S_{T}\right)= & f\left(S_{t}\right)+f^{\prime}\left(S_{t}\right)\left(S_{T}-S_{t}\right) \\
& +\int_{S_{t}}^{S_{T}} f^{\prime \prime}(K)\left(S_{T}-K\right) d K
\end{aligned}
$$

$$
\begin{aligned}
= & f\left(S_{t}\right)+f^{\prime}\left(S_{t}\right)\left(S_{T}-S_{t}\right) \\
& +\int_{S_{t}}^{S_{T}} f^{\prime \prime}(K)\left(S_{T}-K\right)^{+} d K \\
& +\int_{S_{T}}^{S_{t}} f^{\prime \prime}(K)\left(K-S_{T}\right)^{+} d K .
\end{aligned}
$$

By definition, $\Phi_{R_{t, T-t}}(x)=E_{\pi^{*}}\left(f\left(S_{T}\right)\right)$. Given (A.2), the characteristic function $\Phi_{R_{t, T-t}}(x)$ can then be expressed as

$$
\begin{aligned}
& \Phi_{R_{t, T-t}}(x)=1+ i x\left(\frac{E_{\pi^{*}}\left(S_{T}\right)}{S_{t}}-1\right) \\
&-\left[\int_{S_{t}}^{\infty} \frac{x(x+i)}{K^{2}}\left(\frac{K}{S_{t}}\right)^{i x} E_{\pi^{*}}\left(\left(S_{T}-K\right)^{+}\right) d K\right. \\
&+\int_{0}^{S_{t}} \frac{x(x+i)}{K^{2}}\left(\frac{K}{S_{t}}\right)^{i x} \\
&\left.\times E_{\pi^{*}}\left(\left(K-S_{T}\right)^{+}\right) d K\right] .
\end{aligned}
$$

Under no arbitrage condition, $\Phi_{R_{t, T-t}}(x)$ can be further given by

$$
\begin{gathered}
\Phi_{R_{t, T-t}}(x)=1+i x\left[e^{(r-q)(T-t)}-1\right] \\
-\left[\int_{S_{t}}^{\infty} \frac{x(x+i)}{K^{2}}\left(\frac{K}{S_{t}}\right)^{i x} e^{r(T-t)} C_{t}^{E}(T ; K) d K\right. \\
+\int_{0}^{S_{t}} \frac{x(x+i)}{K^{2}}\left(\frac{K}{S_{t}}\right)^{i x} \\
\left.\quad \times e^{r(T-t)} P_{t}^{E}(T ; K) d K\right] .
\end{gathered}
$$

Given (A.4), the $j$ th order RNM, $m_{t, T-t}(j)$, defined as $E_{\pi^{*}}\left(\left[\log \left(R_{T-t}\right)\right]^{j}\right)$, can then be calculated by $\left.\left(1 / i^{j}\right)\left(d^{j}\left[\Phi_{R_{t, T-t}}(x)\right] / d x^{j}\right)\right|_{x=0}(j=1,2,3,4, \ldots, J)$.

\section{B. Proof of Theorem 3}

Denote the characteristic functions of the $(T-t)$-period return, $\log \left(R_{t, T-t}\right)=\log \left(S_{T} / S_{t}\right)$ and the $\tau$-period return, $\log \left(R_{t, \tau}\right)=\log \left(S_{t+\tau} / S_{t}\right)$, as $\Phi_{R_{t, T-t}}(x)=E_{\pi^{*}}\left(e^{i x \log \left(R_{t, T-t}\right)}\right)$ and $\Phi_{R_{t, \tau}}(x)=E_{\pi^{*}}\left(e^{i x \log \left(R_{t, \tau}\right)}\right)$, respectively. Then, by the 
assumption that the $\tau$-period returns are independent under the risk-neutral measure $\widehat{\pi}^{*}$,

$$
\begin{aligned}
& \Phi_{R_{t, T-t}}(x) \\
& =E_{\pi^{*}}\left(e^{i x \log \left(R_{T-t}\right)}\right)=E_{\pi^{*}}\left[\left(\frac{S_{T}}{S_{t}}\right)^{i x}\right] \\
& =E_{\pi^{*}}\left[\left(\frac{S_{T}}{S_{T-\tau}}\right)^{i x}\left(\frac{S_{T-\tau}}{S_{T-2 \tau}}\right)^{i x}\left(\frac{S_{T-2 \tau}}{S_{T-3 \tau}}\right)^{i x} \cdots\left(\frac{S_{\tau}}{S_{t}}\right)^{i x}\right] \\
& =\left[E_{\pi^{*}}\left(R_{t, \tau}^{i x}\right)\right]^{N},
\end{aligned}
$$

or, equivalently, $\Phi_{R_{t, \tau}}(x)=\left[\Phi_{R_{t, T-t}}(x)\right]^{1 / N}$.

As $m_{t, \tau}(1)=\left.(1 / i)\left(d\left[\Phi_{R_{t, \tau}}(x)\right] / d x\right)\right|_{x=0}$, we then have

$$
\begin{aligned}
m_{t, \tau}(1) & =\left.\frac{1}{i} \frac{d\left[\Phi_{R_{t, \tau}}(x)\right]}{d x}\right|_{x=0} \\
& =\left.\left.\frac{1}{i} \frac{1}{N}\left\{\left[\Phi_{R_{t, T-t}}(x)\right]^{(1 / N)-1}\right\}\right|_{x=0} \frac{d\left[\Phi_{R_{t, T-t}}(x)\right]}{d x}\right|_{x=0} \\
& =\frac{m_{t, T-t}(1)}{N} .
\end{aligned}
$$

Repeating the above differentiation procedures, the remaining formulas for $m_{t, \tau}(1), m_{t, \tau}(2), m_{t, \tau}(3)$, and $m_{t, \tau}(4)$ are immediately obtained.

\section{Calculation of Integrals for Moments}

Consider

$$
\begin{gathered}
m_{t, T-t}(1)=e^{(r-q)(T-t)} \\
-e^{r(T-t)}\left[\int_{S_{t}}^{\infty} \frac{1}{K^{2}} C_{t}^{A}(T ; K) d K\right. \\
\left.\quad+\int_{0}^{S_{t}} \frac{1}{K^{2}} P_{t}(T ; K) d K\right]-1, \\
m_{t, T-t}(2)=2 e^{r(T-t)}\left[\int_{S_{t}}^{\infty} \frac{1-\ln \left(K / S_{t}\right)}{K^{2}} C_{t}^{A}(T ; K) d K\right. \\
\left.\quad+\int_{0}^{S_{t}} \frac{1-\ln \left(K / S_{t}\right)}{K^{2}} P_{t}(T ; K) d K\right],
\end{gathered}
$$

where $P_{t}(T ; K)=C_{t}^{A}(T ; K)+K e^{-r(T-t)}-S_{t} e^{-q(T-t)}$.

We only discuss the calculation of the integrals in the first-order moment: $\int_{S_{t}}^{\infty}\left(1 / K^{2}\right) C_{t}^{A}(T ; K) d K$ and $\int_{0}^{S_{t}}\left(1 / K^{2}\right) P_{t}(T ; K) d K$. The remaining integrals appearing in other moment equations can be solved analogously.

\section{C.1. Calculation of Integrals}

C.1.1. $\int_{S_{t}}^{\infty}\left(1 / K^{2}\right) C_{t}^{A}(T ; K) d K$. First, the interval of this integration $\left[S_{t}, \infty\right)$ is divided into three subintervals: $\left[S_{t}, K_{\max }\right]$, $\left[K_{\max }, K_{\infty}\right)$, and $\left[K_{\infty}, \infty\right)$, where $K_{\max }$ is the maximum available strike price in the given data, whereas $K_{\infty}$ is a much larger number so that a call option with strike prices in $\left[K_{\infty}, \infty\right)$ is valueless. This study sets $K_{\infty}$ equal to $5 K_{\max }$.

Second, a numerical integration method, the trapezoidal rule, is employed to compute the integrals with the first two intervals:

$$
\begin{aligned}
\int_{S_{t}}^{K_{\max }} & \frac{1}{K^{2}} C_{t}^{A}(T ; K) d K \\
\quad \approx & \frac{1}{2}\left[\sum_{i=1}^{m}\left(\frac{1}{K_{i-1}^{2}} C_{t}^{A}\left(T ; K_{i-1}\right)+\frac{1}{K_{i}^{2}} C_{t}^{A}\left(T ; K_{i}\right)\right) \Delta K\right],
\end{aligned}
$$

where $\Delta K=\left(K_{\max }-S_{t}\right) / m, K_{i}=S_{t}+i \Delta K$, for $i \in[0, m] . m$ denotes the number of nonoverlapped subintervals of equal length and $m=80$ in this study ${ }^{20}$.

In a similar spirit, the integral with the second subinterval is given as

$$
\begin{aligned}
& \int_{K_{\max }}^{K_{\infty}} \frac{1}{K^{2}} C_{t}^{A}(T ; K) d K \\
& \quad \approx \frac{1}{2}\left[\sum_{i=1}^{m}\left(\frac{1}{K_{i-1}^{\prime 2}} C_{t}^{A}\left(T ; K_{i-1}^{\prime}\right)+\frac{1}{K_{i}^{\prime 2}} C_{t}^{A}\left(T ; K_{i}^{\prime}\right)\right) \Delta K^{\prime}\right],
\end{aligned}
$$

where $\Delta K^{\prime}=\left(K_{\infty}-K_{\max }\right) / m, K_{i}^{\prime}=K_{\max }+i \Delta K^{\prime}$ for $i \in[0, m]$. Given the negligible integrand in interval $\left[K_{\infty}, \infty\right)$, we obtain

$$
\begin{aligned}
\int_{S_{t}}^{\infty} & \frac{1}{K^{2}} C_{t}^{A}(T ; K) d K \\
\approx & \frac{1}{2}\left[\sum_{i=1}^{m}\left(\frac{1}{K_{i-1}^{2}} C_{t}^{A}\left(T ; K_{i-1}\right)+\frac{1}{K_{i}^{2}} C_{t}^{A}\left(T ; K_{i}\right)\right) \Delta K\right] \\
& +\frac{1}{2}\left[\sum_{i=1}^{m}\left(\frac{1}{K_{i-1}^{\prime 2}} C_{t}^{A}\left(T ; K_{i-1}^{\prime}\right)+\frac{1}{K_{i}^{\prime 2}} C_{t}^{A}\left(T ; K_{i}^{\prime}\right)\right) \Delta K^{\prime}\right] .
\end{aligned}
$$

C.1.2. $\int_{0}^{S_{t}}\left(1 / K^{2}\right) P_{t}(T ; K) d K$. This integral can be computed by repeating the above steps. Note that the three subintervals are $\left[0, K_{0}\right],\left[K_{0}, K_{\min }\right]$, and $\left[K_{\min }, S_{t}\right]$, where $K_{\min }$ is the minimum available strike price in the given data, whereas $K_{0}$ is a very smaller number so that a put option with strike prices 
in $\left[0, K_{0}\right]$ is valueless. In this study, $K_{0}$ is set to the value of $0.2 K_{\min }$. We then have

$$
\begin{aligned}
\int_{0}^{S_{t}}( & \left.\frac{1}{K^{2}}\right) P_{t}(T ; K) d K \\
\approx & \frac{1}{2}\left[\sum_{i=1}^{m}\left(\frac{1}{K_{i-1}^{2}} P_{t}\left(T ; K_{i-1}\right)+\frac{1}{K_{i}^{2}} P_{t}\left(T ; K_{i}\right)\right) \Delta K\right] \\
& +\frac{1}{2}\left[\sum_{i=1}^{m}\left(\frac{1}{K_{i-1}^{\prime 2}} P_{t}\left(T ; K_{i-1}^{\prime}\right)+\frac{1}{K_{i}^{\prime 2}} P_{t}\left(T ; K_{i}^{\prime}\right)\right) \Delta K^{\prime}\right],
\end{aligned}
$$

where $\Delta K=\left(K_{\min }-K_{0}\right) / m, \Delta K^{\prime}=\left(S_{t}-K_{\min }\right) / m, K_{i}=$ $K_{0}+i \Delta K$, and $K_{i}^{\prime}=K_{\min }+i \Delta K^{\prime}$ for $i \in[0, m]$.

C.2. Curving-Fitting for Unavailable Option Prices. As shown from the above integrals, the required strike prices are beyond the range of the available data. The option prices corresponding to such strike prices need to be inferred from the given option prices. A curve-fitting method is adapted to handle this restriction by first constructing a set of implied volatilities and then inferring the required set of option prices. The operational steps are as follows.

(1) Calculate implied volatilities using the Black-Scholes formula based on the given set of option (either simulated or observed from the market) with trading date $t$ and expiry date $T^{21}$.

(2) Use a cubic spline function to interpolate the implied volatilities and infer implied volatilities at $K_{i}$ and $K_{i}^{\prime}$ located in $\left[S_{t}, K_{\max }\right]$ or $\left[K_{\min }, S_{t}\right]$ from the fitted function.

(3) Inversely map the inferred volatilities, again using the Black-Scholes formula, to obtain the required option prices $C_{t}^{A}(T ; K), C_{t}^{A}\left(T ; K^{\prime}\right), P_{t}(T ; K)$, and $P_{t}\left(T ; K^{\prime}\right)$ over the intervals of $\left[S_{0}, K_{\max }\right]$ and $\left[K_{\min }, S_{t}\right]$.

(4) Apply two implied volatilities at the truncated endpoints $K_{0}$ and $K_{\infty}$ to the intervals $\left[K_{\max }, K_{\infty}\right.$ ] and $\left[K_{0}, K_{\min }\right]$ to extrapolate the option prices.

Recall that in the simulation experiment, we generate a sample of 8 call options to estimate the RNMs. The other options required by calculating the integrals are referred to by the method discussed above.

\section{Conflict of Interests}

The authors declare that there is no conflict of interests regarding the publication of this paper.

\section{Acknowledgments}

The authors thank Wolfgang Buehler, David Feldman, Robert Kohn, Donald Lien, Jun Liu, and Qiang Liu for their valuable discussions and comments as well as seminar participants at the 2011 FMA annual meeting, the 24th AFB conference, and
2013 CFR conference. Xiaoke Xie is thanked for proofreading this paper. The authors are truly grateful to the anonymous reviewers for their helpful comments. The editorial support from Editor Fenghua Wen is sincerely appreciated without any reservation. This work is supported by the National Natural Science Foundation of China (no. 71301132), by the Fundamental Research Funds for the Central Universities, by the Sichuan Provincial Department of Education Research Project (14ZB0448), and by the ARC Discovery Grant (DP120104014).

\section{Endnotes}

1. See, for example, Jarrow and Rudd [15], Bahra [16], AïtSahalia and Lo [17], and Jackwerth [18] for a comprehensive review. See, for example, Bates [19], Grundy [20], Jackwerth and Rubinstein [21], Melick and Thomas [22], Bakshi and Madan [23], Britten-Jones and Neuberger [24], Bakshi et al. [9], Jiang and Tian [25], Kang and Kim [26], Chang et al. [27], and Diavatopoulos et al. [28] for methodologies and application issues.

2. For example, Kang and Kim [26] report that option prices exactly reflect market expectations of abnormal or rare future asset market events and return distributions. Jiang and Tian [25] show that the implied volatility simply from OTM European call options subsumes all information contained in Black-Scholes implied volatility and past realized volatility (Chiras and Manaster [29] and Day and Lewis [30]).

3. Bakshi et al. [9] use a Taylor expansion to obtain the expressions for the first four moments, which can be proved and extended by using the characteristic function instead.

4. In this study, the underlying asset is set to pay no dividend in the simulation experiments and in the empirical section, any IBM call options violating the principle of not exercising early (see footnote 18) are removed. So the call options in both cases cannot be exercised early.

5. In the simulations, $q=0$. In the empirical investigation, we find that almost all the IBM options satisfy the condition of discrete dividends.

6. Many empirical investigations (e.g., Zivney [31] and Poteshman and Serbin [32]) show that call options with such low moneyness $\left(S_{t} / K\right)$ are not being exercised before expiration in most cases. Broadie et al. [33] show empirically that for American calls of OEX 100 index options, most of the early exercises occur during the last few days prior to the expiration month only when the moneyness is close to 1 .

7. Note that the idea of fitting option prices is suggested, in particular, by Jiang and Tian [25], who provide the theoretical bounds on truncation errors. In our study, the interval of integration $\left[0, S_{t}\right]$ is split into two subintervals, $\left[0, K_{\min }\right]$ and $\left[K_{\min }, S_{t}\right]$, and the other interval $\left[S_{t},+\infty\right)$ is split into $\left[S_{t}, K_{\max }\right]$ and $\left[K_{\max },+\infty\right)$, where $K_{\min }$ is the minimum value of strike price and 
$K_{\max }$ is the maximum value. For the integrals over the intervals $\left[K_{\min }, S_{t}\right]$ and $\left[S_{t}, K_{\max }\right]$, we use a cubic spline function to interpolate the implied volatilities to obtain the fitted option prices. In addition, we use two constants (endpoint implied volatilities) to extrapolate the option prices for the other two intervals beyond the available range. The extrapolation is truncated at the strike points, denoted as $K_{0}$ and $K_{\infty}$. The first (second) truncation point is very small (large), say, close to zero (an arbitrary large number), so that the corresponding options $C^{A}(T ; K)(P(T ; K))$ with strike prices in the interval $\left[0, K_{0}\right]\left(\left(K_{\infty},+\infty\right)\right)$ are valueless. The integrals over the corresponding intervals therefore tend to be zero. For further details, see Appendix C.2.

8. When approximating the integrals using a numerical integration method, two types of Riemann integral sum are utilized. Specifically, Riemann sums of the left endpoints as well as the right endpoints are first calculated, and their average is then used as an approximation of the required integral. In this study, each of the intervals involved in integration is divided into a number $m$ (e.g., $m=80$ ) of equidistant subintervals (see Appendix C.1 for details).

9. When the empirical distribution is uniform, the Kullback-Leibler information criterion (Kullback and Leibler [34]) is equivalent to the principle of maximum entropy.

10. Note that $R_{t-(I-i+1) \tau, \tau}$ are the historical returns. We recover the RNMs using option data at time $t$ and estimate the RND using the $\tau$-period historical returns starting at time $t-I \tau$ up to time $t$.

11. In our article, $\lambda^{*}$ is calculated via the Nelder-Mead simplex search method by setting the initial value $\lambda_{0}$ equal to the optimal value $\lambda_{0}^{*}$ obtained using quasiNewton method. That is, $\lambda_{0}^{*}$ is first computed via quasiNewton method, by setting initial value to be zero vector and then used as an initial value when calculating $\lambda^{*}$. Theoretically, Nelder-Mead simplex search method is more stable, while the frequently used quasi-Newton method is faster. For discussion about the solution, see Agmon et al. [7] and Mead and Papanicolaou [35].

12. Note that the asset prices in the basis functions should be normalized by the strike price to avoid numerical scaling issues. Moreover, to balance computational time and precision, Stentoft [11] suggests that the Legendre polynomial family with two or three simple polynomial basis functions seems to work better and is computationally less demanding than other orthogonal polynomial families, such as the Laguerre family proposed by Longstaff and Schwartz [5].

13. The dividend is not considered here for the impartiality of comparison.

14. In this simulation experiment, we just compare RMEL method with that of Liu [4] which has no other constraint, since the results not reported here show that RMEL method performs similarly to the methods of
Alcock and Auerswald [6]. The reason for this might be due to that the simulation setting is based on the GBM process so that theoretically the constraints used in those two methods can determine the same risk-neutral distribution for the underlying asset's return.

15. Here $I=365$ is used as did in Liu [4]; we can also choose $I$ more than 365 . The idea is to use the information as recent as possible. If $I$ is too large, for example, over 70,000 historical time series data are required for obtaining the RND in Alcock and Auerswald [6], practically the data from the real market may be outdated or they may be infeasible to be collected.

16. In our empirical investigation discussed in Section 4, the minimum number of options with different strike prices is 8 (with trading date August 21, 2008 and expiration date September 19, 2008) and the maximum number is 22 (e.g., options with trading date December 16, 2008 and expiration date April 17,2009). Further, Buchen and Kelly [8] suggest that it is sufficient even if the number is 3 in their study.

17. Moneyness is defined to be $S_{t} / K$ for call options and $K / S_{t}$ for put options.

18. Several filters are applied to the sample of data prior to conducting our empirical analysis. First, data with market prices less than $\$ 0.05$ are excluded. Second, the prices of put (call) options should theoretically increase (decrease) with strike prices. Data violating this rule are discarded. Third, any call options violating the principle of not exercising early $\left(D_{i} \leq K\left[1-e^{-r\left(t_{i+1}-t_{i}\right)}\right]\right)$, where $D_{i}$ is the dividend payment at time $t_{i}$, see Hull [12, pp.299300], are omitted. Fourth, call options with negative implied Black-Scholes volatility are removed from the sample.

19. In this paper, the MPE is calculated by dividing the estimated price minus the "true" price by the "true" price and multiplying by 100 . It is then averaged over $n=800$ independent simulations and is given as $(1 / n) \sum_{i=1}^{n}\left[\left(c_{i}^{\text {estimated }}-c^{\text {true }}\right) / c^{\text {true }}\right] \times 100$. The MSE is calculated by the mean-squared difference between the estimated price and the "true" price over all the simulations: $(1 / n) \sum_{i=1}^{n}\left(c_{i}^{\text {estimated }}-c^{\text {true }}\right)^{2}$. The MAPE is calculated by dividing the absolute difference between the estimated price and the "true" price by the "true" price and multiplying by 100. It is then averaged over all the simulations and is given as $(1 / n) \sum_{i=1}^{n}\left(\left|c_{i}^{\text {estimated }}-c^{\text {true }}\right| / c^{\text {true }}\right) \times 100$.

20. A value of $m=80$ here is sufficiently large to obtain an accurate approximation of the required integrals (also see Jiang and Tian [25], for another example). We facilitate a comparison by setting that $m=50$ and find no significant difference.

21. Note that the Black-Scholes formula here is merely used as a tool to build a smooth nonlinear relation between volatility and option prices. 


\section{References}

[1] M. Frittelli, "The minimal entropy martingale measure and the valuation problem in incomplete markets," Mathematical Finance, vol. 10, no. 1, pp. 39-52, 2000.

[2] M. Stutzer, "A simple nonparametric approach to derivative security valuation," The Journal of Finance, vol. 51, no. 5, pp. 1633-1652, 1996.

[3] E. T. Jaynes, "On the rationale of maximum-entropy methods," Proceedings of the IEEE, vol. 70, no. 9, pp. 939-952, 1982.

[4] Q. Liu, "Pricing American options by canonical least-squares Monte Carlo," The Journal of Futures Markets, vol. 30, no. 2, pp. 175-187, 2010.

[5] F. A. Longstaff and E. S. Schwartz, "Valuing American options by simulation: a simple least-squares approach," The Review of Financial Studies, vol. 14, no. 1, pp. 113-147, 2001.

[6] J. Alcock and D. Auerswald, "Empirical tests of canonical nonparametric American option-pricing methods," The Journal of Futures Markets, vol. 30, no. 6, pp. 509-532, 2010.

[7] N. Agmon, Y. Alhassid, and R. D. Levine, "An algorithm for finding the distribution of maximal entropy," Journal of Computational Physics, vol. 30, no. 2, pp. 250-258, 1979.

[8] P. W. Buchen and M. Kelly, "The maximum entropy distribution of an asset inferred from option prices," Journal of Financial and Quantitative Analysis, vol. 31, no. 1, pp. 143-159, 1996.

[9] G. Bakshi, N. Kapadia, and D. Madan, "Stock return characteristics, skew laws, and the differential pricing of individual equity options," The Review of Financial Studies, vol. 16, no. 1, pp. 101143, 2003.

[10] P. Brandimarte, Numerical Methods in Finance and Economics: A MATLAB Based Introduction, Statistics in Practice, John Wiley \& Sons, Hoboken, NJ, USA, 2nd edition, 2006.

[11] L. Stentoft, "Assessing the least squares Monte-Carlo approach to American option valuation," Review of Derivatives Research, vol. 7, no. 2, pp. 129-168, 2004.

[12] J. C. Hull, Options, Futures, and Other Derivatives, Pearson Prentice Hall, Upper Saddle River, NJ, USA, 7th edition, 2009.

[13] P. Gray and S. Newman, "Canonical valuation of options in the presence of stochastic volatility," The Journal of Futures Markets, vol. 25, no. 1, pp. 1-19, 2005.

[14] R. M. Dudley, Real Analysis and Probability, vol. 74 of Cambridge Studies in Advanced Mathematics, Cambridge University Press, Cambridge, Mass, USA, 2002.

[15] R. Jarrow and A. Rudd, "Approximate option valuation for arbitrary stochastic processes," Journal of Financial Economics, vol. 10, no. 3, pp. 347-369, 1982.

[16] B. Bahra, "Implied risk-neutral probability density functions from option prices: theory and application," Working Paper 66, Bank of England, 1997, http://ssrn.com/abstract=77429.

[17] Y. Aït-Sahalia and A. W. Lo, "Nonparametric estimation of state-price densities implicit in financial asset prices," The Journal of Finance, vol. 53, no. 2, pp. 499-547, 1998.

[18] J. C. Jackwerth, "Option-implied risk-neutral distributions and implied binomial trees: a literature review," The Journal of Derivatives, vol. 7, no. 2, pp. 66-82, 1999.

[19] D. Bates, "The crash of '87: was it expected? The evidence from options markets," The Journal of Finance, vol. 46, no. 3, pp. 10091044, 1991.

[20] B. D. Grundy, "Option prices and the underlying asset's return distribution," The Journal of Finance, vol. 46, pp. 1045-1069, 1991.
[21] J. C. Jackwerth and M. Rubinstein, "Recovering probability distributions from option prices," The Journal of Finance, vol. 51, no. 5, pp. 1611-1631, 1996.

[22] W. R. Melick and C. P. Thomas, "Recovering an asset's implied PDF from option prices: an application to crude oil during the Gulf crisis," Journal of Financial and Quantitative Analysis, vol. 32, no. 1, pp. 91-115, 1997.

[23] G. Bakshi and D. Madan, "Spanning and derivative-security valuation," Journal of Financial Economics, vol. 55, no. 2, pp. 205-238, 2000.

[24] M. Britten-Jones and A. Neuberger, "Option prices, implied price processes, and stochastic volatility," The Journal of Finance, vol. 55, no. 2, pp. 839-866, 2000.

[25] G. J. Jiang and Y. S. Tian, "The model-free implied volatility and its information content," The Review of Financial Studies, vol. 18, no. 4, pp. 1305-1342, 2005.

[26] B. J. Kang and T. S. Kim, "Option-implied risk preferences: an extension to wider classes of utility functions," Journal of Financial Markets, vol. 9, no. 2, pp. 180-198, 2006.

[27] C.-C. Chang, P.-F. Hsieh, and Y.-H. Wang, "Information content of options trading volume for future volatility: evidence from the Taiwan options market," Journal of Banking and Finance, vol. 34, no. 1, pp. 174-183, 2010.

[28] D. Diavatopoulos, J. S. Doran, A. Fodor, and D. R. Peterson, "The information content of implied skewness and kurtosis changes prior to earnings announcements for stock and option returns," Journal of Banking and Finance, vol. 36, no. 3, pp. 786802, 2012.

[29] D. P. Chiras and S. Manaster, "The information content of option prices and a test of market efficiency," Journal of Financial Economics, vol. 6, no. 2-3, pp. 213-234, 1978.

[30] T. E. Day and C. M. Lewis, "Stock market volatility and the information content of stock index options," Journal of Econometrics, vol. 52, no. 1-2, pp. 267-287, 1992.

[31] T. L. Zivney, "The value of early exercise in option prices: an empirical investigation," Journal of Financial and Quantitative Analysis, vol. 26, pp. 129-138, 1991.

[32] A. M. Poteshman and V. Serbin, "Clearly irrational financial market behavior: evidence from the early exercise of exchange traded stock options," The Journal of Finance, vol. 58, no. 1, pp. 37-70, 2003.

[33] M. Broadie, J. Detemple, E. Ghysels, and O. Torrès, "Nonparametric estimation of American options' exercise boundaries and call prices," The Journal of Economic Dynamics and Control, vol. 24, no. 11-12, pp. 1829-1857, 2000.

[34] S. Kullback and R. A. Leibler, "On information and sufficiency," Annals of Mathematical Statistics, vol. 22, pp. 79-86, 1951.

[35] L. R. Mead and N. Papanicolaou, "Maximum entropy in the problem of moments," Journal of Mathematical Physics, vol. 25, no. 8, pp. 2404-2417, 1984. 


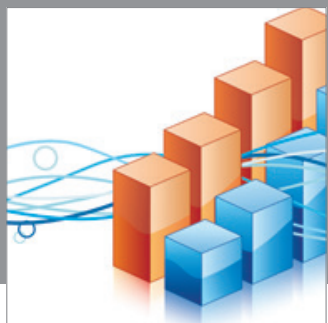

Advances in

Operations Research

mansans

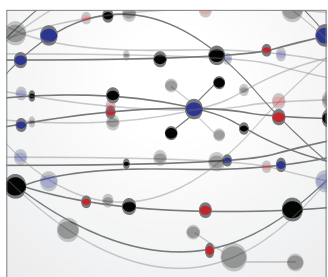

The Scientific World Journal
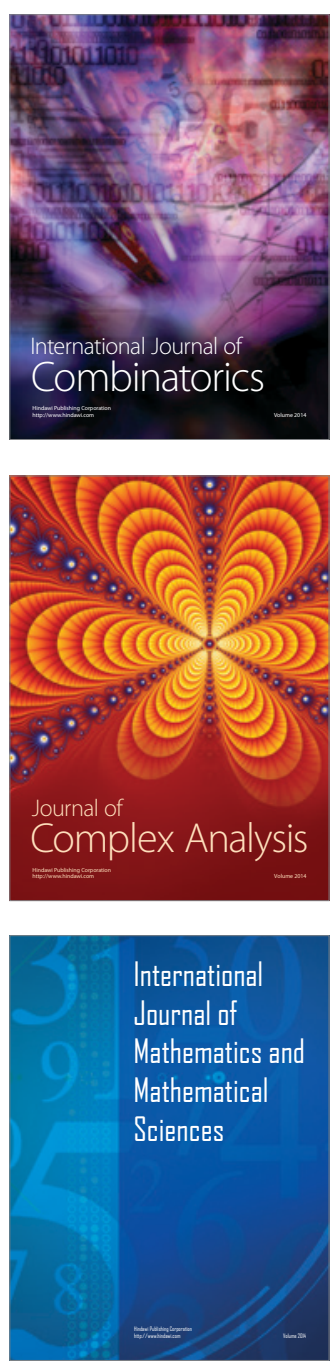
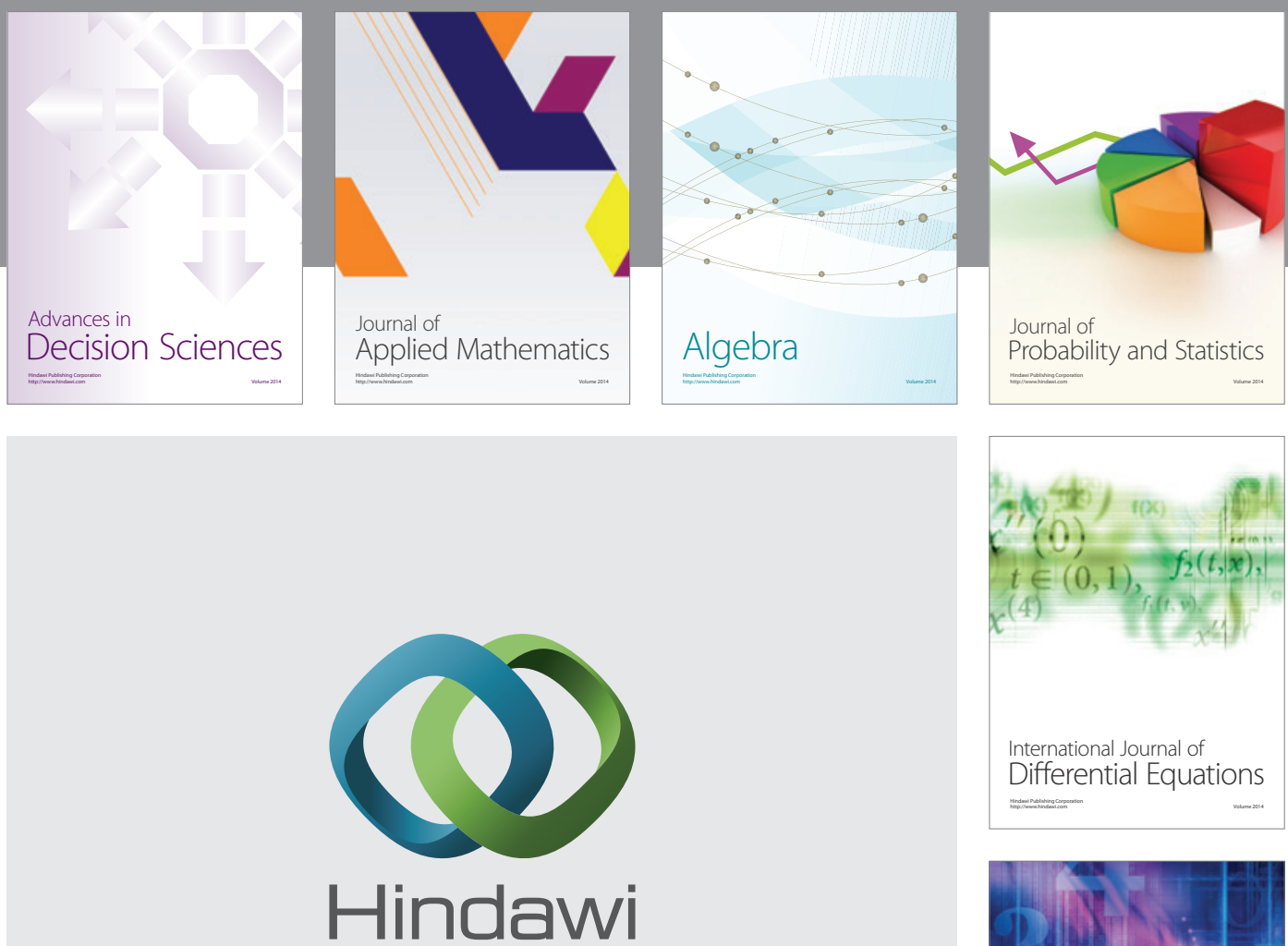

Submit your manuscripts at http://www.hindawi.com
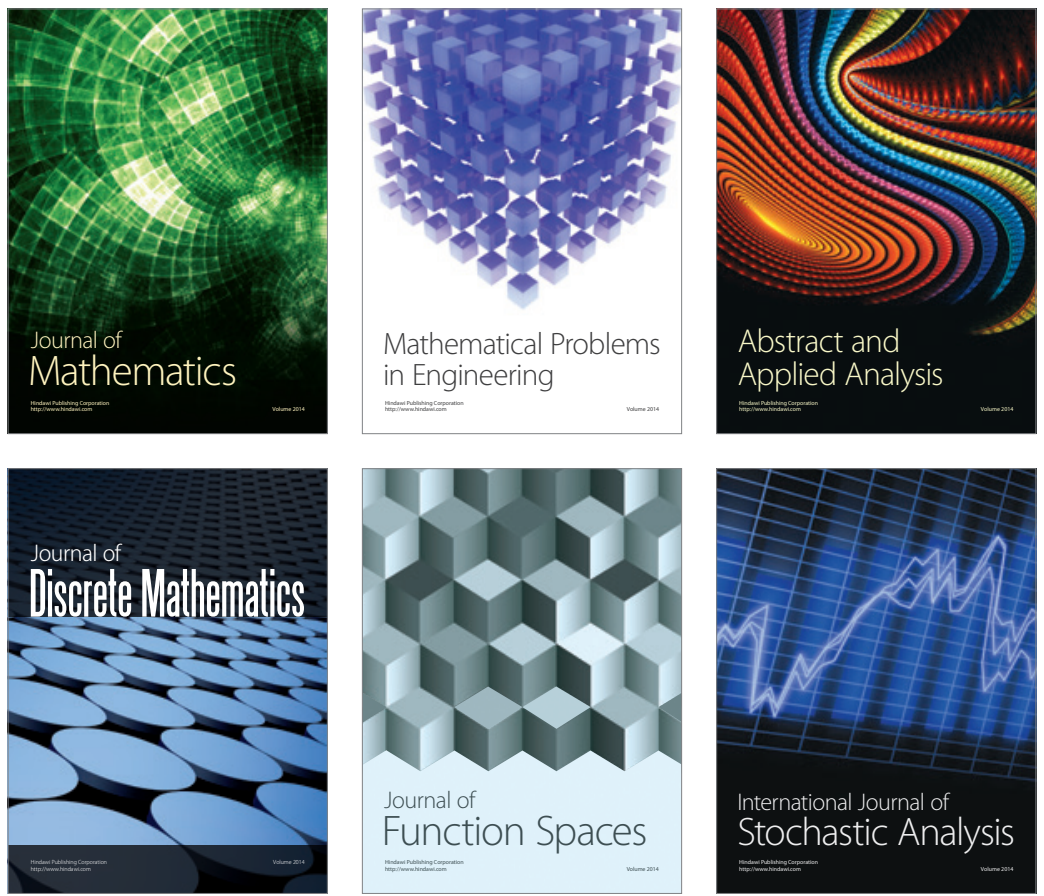

Journal of

Function Spaces

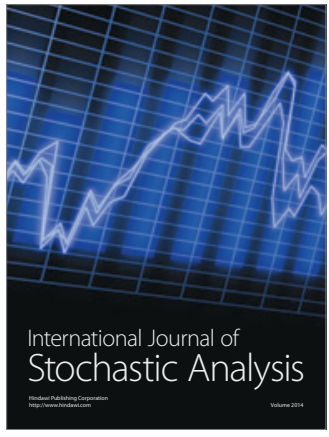

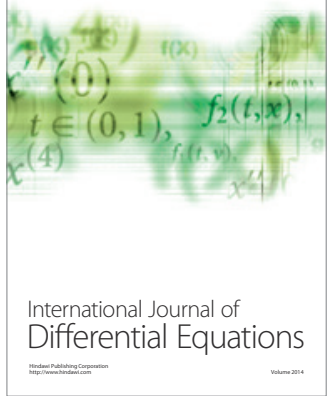
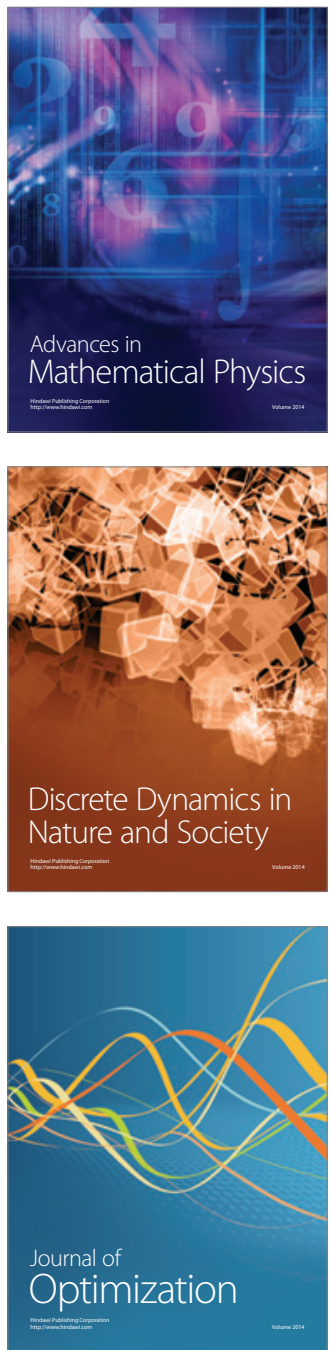